\title{
Aerobic Exercise and Neurocognitive Performance: A Meta-Analytic Review of Randomized Controlled Trials
}

Patrick J. Smith, MA, James A. Blumenthal, PhD, Benson M. Hoffman, PhD, Harris Cooper, PhD, Timothy A. Strauman, PhD, Kathleen Welsh-Bohmer, PhD, Jeffrey N. Browndyke, PhD, ANd Andrew Sherwood, PhD

\begin{abstract}
Objectives: To assess the effects of aerobic exercise training on neurocognitive performance. Although the effects of exercise on neurocognition have been the subject of several previous reviews and meta-analyses, they have been hampered by methodological shortcomings and are now outdated as a result of the recent publication of several large-scale, randomized, controlled trials (RCTs). Methods: We conducted a systematic literature review of RCTs examining the association between aerobic exercise training on neurocognitive performance between January 1966 and July 2009. Suitable studies were selected for inclusion according to the following criteria: randomized treatment allocation; mean age $\geq 18$ years of age; duration of treatment $>1$ month; incorporated aerobic exercise components; supervised exercise training; the presence of a nonaerobic-exercise control group; and sufficient information to derive effect size data. Results: Twenty-nine studies met inclusion criteria and were included in our analyses, representing data from 2049 participants and 234 effect sizes. Individuals randomly assigned to receive aerobic exercise training demonstrated modest improvements in attention and processing speed $(\mathrm{g}=0.158 ; 95 \%$ confidence interval $[\mathrm{CI}] ; 0.055-0.260$; $p=.003)$, executive function $(\mathrm{g}=0.123 ; 95 \% \mathrm{CI}, 0.021-0.225 ; p=.018)$, and memory $(\mathrm{g}=0.128 ; 95 \% \mathrm{CI}, 0.015-0.241$; $p=.026)$. Conclusions: Aerobic exercise training is associated with modest improvements in attention and processing speed, executive function, and memory, although the effects of exercise on working memory are less consistent. Rigorous RCTs are needed with larger samples, appropriate controls, and longer follow-up periods. Key words: cognitive performance, aerobic exercise, neuropsychological performance, executive function, randomized controlled trial, meta-analysis.
\end{abstract}

ITT $=$ intention-to-treat; $\mathbf{R C T}=$ randomized controlled trial

\section{INTRODUCTION}

Ctrategies to enhance neurocognitive functioning have important public health implications as subclinical neurocognitive deficits are associated with increased risk of neurocognitive impairment (1), dementia (2), and mortality (3-7), independent of traditional risk factors. One such strategy that has gained increased attention is the use of aerobic exercise to improve neurocognitive functioning $(8-12)$. Although the value of exercise has been critically examined in review articles (13) and meta-analytic syntheses (8-11), there has been a lack of agreement as to the magnitude of improvement in neurocognitive function associated with physical activity interventions. The current lack of consensus is due to differences in the evaluation of study methodologies, studies included in the analyses, data analytic approaches, and in the classification of various neurocognitive measures.

Cross-sectional studies have shown that physically active individuals tend to exhibit better neurocognitive function relative to inactive individuals (13-22). Prospective observational studies have reported similar findings, demonstrating that individuals who maintain greater levels of physical activity show improvements in neurocognitive function relative to their sedentary counterparts (1,23-28). However, randomized

From the Department of Psychiatry and Behavioral Sciences (P.J.S., J.A.B., B.M.H., K.W.-B., J.N.B., A.S.), Duke University Medical Center, Durham, North Carolina; and the Department of Psychology and Neuroscience (H.C., T.A.S.), Duke University, Durham, North Corolina.

The research was supported, in part, by Grants MH 49679 and HL08066401A1 from the National Institutes of Health and Grant M01-RR-30 from the General Clinical Research Center Program, National Center for Research Resources, National Institutes of Health (J.A.B.).

Address correspondence and reprint requests to Patrick Smith, Box 3119, Department of Psychiatry and Behavioral Sciences, Duke University Medical Center, Durham, NC 27710. E-mail: Smith562@mc.duke.edu

Received for publication March 23, 2009; revision received December 3, 2009. DOI: 10.1097/PSY.0b013e3181d14633 trials have provided inconsistent results, with some reporting cognitive gains $(29,30)$ and others equivocal findings (31). Meta-analytic reviews of randomized controlled trials (RCTs) have also reported great variation in the magnitude of improvement in neurocognition associated with aerobic exercise (10-12), with some meta-analyses reporting moderate cognitive gains $(9,10)$ and others reporting more modest improvements $(8,11,32)$.

In several recent meta-analyses, including a Cochrane review (11), it was concluded that current data are insufficient to show that improvements in neurocognitive function associated with physical activity are due to improved cardiovascular fitness, and that larger studies are necessary $(11,32)$. However, since the publication of this review, there have been several large-scale RCTs examining this relationship (30,31,33,34). In addition, although one previous systematic review (12) examined the effects of various forms of physical activity on boosting cognitive function (primarily general orientation) among individuals with dementia, no reviews have combined data from trials attempting to prevent dementia among vulnerable populations (i.e., individuals with cognitive impairment). The Cochrane review was limited, in this sense, as persons with neurocognitive impairments (e.g., mild cognitive impairment [MCI] and depression) were excluded (11). Furthermore, previous meta-analyses examining this relationship may have been influenced by the inclusion of two relatively large studies reporting substantial treatment effects that were not truly randomized $(9,35,36)$, which may have overly influenced the reported effects. Therefore, we conducted a metaanalysis that included the most recent exercise intervention trials and addressed several issues including: 1) the effects of aerobic exercise training on specific domains of neurocognitive performance, including attention and processing speed, executive function, working memory, and memory; 2) the influence of specific dimensions of the exercise prescription, such as the mode, duration, and intensity of the exercise 
intervention; and 3) the issue of individual differences in response to exercise training, with a focus on baseline, preexercise level of cognitive functioning as a potential moderator of exercise effects (i.e., we compared individuals with MCI to cognitively intact samples), as well as the age of study participants.

\section{METHODS}

To determine the effects of aerobic exercise interventions on neurocognitive status, an extensive literature search was conducted, using the following databases between January 1966 and July 2009: MEDLINE, Pubmed, EMBASE, Gateway, CENTRAL, PsycINFO, Dissertation Abstracts International, Educational Research in Completion (ERIC), Sports Discus, Cochrane Register, PEDRO, Ageline, and CINAHL. The following search terms were used: cogniti*, cognitive performance, age*, elderly, mental performance, and neuropsychological in combination with fitness, aerobic, cardiovascular, $\mathrm{VO}_{2}$, and physical activity. Additional titles were identified by a manual search of relevant journals and by identification of references included in previous meta-analyses. Unpublished dissertations and conference papers were also obtained, when possible.

Suitable studies were selected for inclusion according to the following criteria: 1) randomized treatment allocation; 2) mean age $\geq 18$ years of age and nondemented; 3) duration of treatment $>1$ month; 4) involved aerobic exercise training (e.g., brisk walking, biking, or jogging). Age 18 years was selected as a lower age limit to control for developmental age differences in cortical thickness and myelination, which stabilize around the second decade of life (37). Studies utilizing walking interventions that were not aerobic were not included (e.g., slow walking with frequent breaks) to ensure that included trials incorporated some aerobic exercise component. Additional inclusion criteria included 5) the presence of a control group that did not engage in aerobic exercise; and 6) sufficient information to derive an estimate of effect size (ES).

After initial identification and retrieval of studies, several were found to be quasirandomized studies (36) or used case-control methodologies $(15,36,38-44)$, were of insufficient duration to include (45-47), were found not to be nonrandomized based on personal communication with the trial's principal investigator (36), or did not utilize a nonaerobic exercise control group $(48,49)$. Another trial was conducted among adolescents and was, therefore, excluded (50). Several trials utilized "dual-task" interventions (e.g., walking and talking) (51-53) or balance and strength-training $(54,55)$ and were, therefore, not included as it could not be ascertained whether exercise was of sufficient intensity to produce aerobic changes. Several trials were not included because they utilized physical activity interventions with exclusively nonaerobic exercise components among individuals with dementia $(52,56-$ 65). The few studies utilizing walking interventions were either explicitly nonaerobic (58) or allowed residents with limited mobility (e.g., using walkers) to rest as needed (52), thereby limiting their generalizability to more healthy samples. Accordingly, these studies were excluded from the current analyses. For two trials in which the method of randomization was unclear $(39,66)$, we attempted to contact the respective authors and were able to confirm that one trial followed a true randomization scheme (39). Results were unchanged when the remaining study was excluded and we, therefore, included this trial in all analyses (66).

\section{Assessment of Study Quality}

Two raters (P.J.S., B.M.H.) independently extracted information from each article, using an identical review protocol, which included study identifiers (e.g., authors' names, year of publication, publishing journal), duration of treatment, intensity of exercise, modality of exercise, blinding of assessment personnel to treatment status, during assessments, intention-to-treat (ITT) analyses, and time of follow-up assessment. ESs were assessed independently. Interrater reliability was assessed for the outcome domains in question (i.e., in each cognitive domain as well as for study characteristics). For all areas, interrater reliability was found to be excellent $(r>.90$; Cohen's $\kappa=0.75)$.

\section{Data Analysis}

Neuropsychological test results were classified according to the cognitive domains described by Lezak and colleagues (67). We considered neurocognitive tests that could be classified in the following categories: attention and processing speed (the sustained focus of cognitive resources with selective concentration and rapid processing of information $(67,68)$, executive function (a set of cognitive skills responsible for the planning, initiation, sequencing, and monitoring of complex, goal-directed behavior), working memory (short-term storage and manipulation of information), and declarative memory (retention, recollection, and recognition of previously encountered information, hereafter referred to only as "memory"). We considered including "complex processing speed" as a measure of executive function as in previous analyses (9), but results were unchanged regardless of the classification of this test.

Analyses were conducted, using Comprehensive Meta Analysis software (Biostat, Englewood, New Jersey). Data were analyzed, using both fixed and random effects models and Cohen's G for between-group differences (69). Fixed effects analysis assumes that all studies are drawn from the same population, such that differences in treatment effects across studies are attributed to sampling and methodological variability (i.e., error variance). In contrast, random effects analysis allows for the possibility that studies are drawn from different populations, such that differences across studies may be due to unidentified sources of variation and provides a more conservative estimate of treatment effects (70). However, because results did not differ between fixed and random effects analyses and because random effects are generally recommended for examining treatment effects in meta-analytic studies (70), we have presented the random effects findings only. In trials reporting multiple effect sizes within the same neurocognitive domain, data were collapsed by averaging all ESs within each neurocognitive domain for each study, such that each study produced no more than one ES per domain. For the purposes of the study quality analyses, treatment effects were collapsed for each study for all neurocognitive domains. In addition, two trials in our literature search produced multiple publications in either peerreviewed journals $(71-73)$ or book chapters $(74,75)$ that were combined for the purposes of analysis. Homogeneity of treatment effects was assessed, using the Q statistic. Three trials collected neurocognitive data at multiple time points in which participants continued to receive treatment $(30,73,76)$. However, in only one study were the effects of treatment uncontaminated by crossover between groups (30). For this study only (30), we chose data from the longest follow-up assessment for inclusion in our analyses, although results were unchanged when other time points were examined.

Exploratory sensitivity analyses $(77,78)$ were conducted to investigate sample characteristics that may have moderated the effects of treatment on neurocognitive outcomes. Specifically, three trial characteristics were examined: duration, intensity, and mode of exercise intervention. We also examined two important methodological characteristics associated with methodological quality: blinding of assessors of neurocognitive outcomes and use of ITT analyses. As an additional analysis, we examined whether treatment effects varied by cognitive status of participants at baseline (i.e., "nonimpaired" or MCI; patients with dementia [Alzheimer's disease] were excluded) and age of study participants.

\section{RESULTS}

Our initial literature search yielded 5538 potentially relevant studies, 68 of which were retrieved for full-text review. Twenty-nine studies incorporating data from 2049 participants met inclusion criteria and were included in the present analyses (Table 1), including data for 1024 experimental participants and 997 controls. Two hundred thirty-four ESs were available for analysis. Trials ranged in duration from 6 weeks (79) to 18 months (30). As shown in Table 1, the primary exercise modality was brisk walking and/or jogging and control groups were typically assigned to a wait-list control, although 
AEROBIC EXERCISE AND NEUROCOGNITION

TABLE 1. Randomized Controlled Trials Examining the Effect of Aerobic Exercise on Neurocognitive Function

\begin{tabular}{|c|c|c|c|c|c|}
\hline Author / Year & Sample & Intervention & Instruments & $\begin{array}{l}\text { Methodological } \\
\text { Characteristics }\end{array}$ & Hedge's G \\
\hline Bakken, 2001 (103) & $\begin{array}{l}15, \text { older adults, ages } \\
72 \text { to } 91\end{array}$ & $\begin{array}{l}\text { Duration: } 8 \text { wks } \\
\text { Frequency: } 30 \text { min, 3/wk } \\
\text { Intensity: - - - } \\
\text { Combined Strength } \\
\text { Training: Y } \\
\mathrm{MCl}: \mathrm{N}\end{array}$ & $\begin{array}{l}\text { Imaging (Verbal Fluency), Visual } \\
\text { Discrimination, Raven's } \\
\text { Progressive Matrices, Short-Term } \\
\text { Retention, Addition, Perception of } \\
\text { Ambiguous Stimuli }\end{array}$ & $\begin{array}{l}\text { Attrition: } 0 \% \\
\text { ITT: } N \\
\text { Blinding: } N\end{array}$ & $\mathrm{AT}=.169$ \\
\hline $\begin{array}{l}\text { Blumenthal, } 1989(72) \\
\quad \& \text { Madden, } 1989(71)\end{array}$ & $\begin{array}{l}101, \text { sedentary, ages } \\
60 \text { to } 83\end{array}$ & $\begin{array}{l}\text { Duration: } 16 \mathrm{wks}^{*} \\
\text { Frequency: } 40 \mathrm{~min}, 3 / \mathrm{wk} \\
\text { Intensity: } 70 \% \text { HRR } \\
\text { Combined Strength } \\
\text { Training: } \mathrm{N} \\
\mathrm{MCl}: \mathrm{N}\end{array}$ & $\begin{array}{l}\text { Finger Tapping, Benton Revised } \\
\text { Visual Retention Test, Digits } \\
\text { Forward, Digits Backward, } \\
\text { Selective Reminding Test, Randt } \\
\text { Memory Test - Short Story, TMT- } \\
\text { B, Digit Symbol, Ruff } 2 \text { \& } 7 \text { Test, } \\
\text { Stroop Color, Stroop Color-Word } \\
\text { Interference, Nonverbal Fluency } \\
\text { Test, Verbal Fluency Test }\end{array}$ & $\begin{array}{l}\text { Attrition: } 8 \% \\
\text { ITT:Y } \\
\text { Blinding: } Y\end{array}$ & $\begin{array}{l}\mathrm{AT}=.218 \\
\mathrm{EX}=-.025 \\
\mathrm{WM}=.114 \\
\mathrm{ME}=-.066\end{array}$ \\
\hline Emery, 1990 (110) & $\begin{array}{l}\text { 48, "inner-city } \\
\text { cohort", ages } 61 \text { to } \\
86\end{array}$ & $\begin{array}{l}\text { Duration: } 12 \text { wks } \\
\text { Frequency: } 60 \mathrm{~min}, 3 / \mathrm{wk} \\
\text { Intensity: } 70 \% \mathrm{HRR} \\
\text { Combined Strength } \\
\text { Training: } \mathrm{Y} \\
\mathrm{MCl} \text { : N }\end{array}$ & $\begin{array}{l}\text { Digit Symbol, Digit Span, Word } \\
\text { Copy, Number Copy }\end{array}$ & $\begin{array}{l}\text { Attrition: } 10 \% \\
\text { ITT: N } \\
\text { Blinding: N }\end{array}$ & $\begin{array}{l}\mathrm{AT}=.028 \\
\mathrm{EX}=-.043 \\
\mathrm{WM}=.023\end{array}$ \\
\hline Emery, 1998 (111) & $\begin{array}{l}79, \text { with stable COPD, } \\
\text { age range not } \\
\text { reported } M=67\end{array}$ & $\begin{array}{l}\text { Duration: } 10 \text { wks }^{*} \\
\text { Frequency: } 45 \text { min, 3/wk } \\
\text { Intensity: - - - } \\
\text { Combined Strength } \\
\text { Training: } \mathrm{Y} \\
\mathrm{MCl}: \mathrm{N}\end{array}$ & $\begin{array}{l}\text { Verbal Fluency, Digit Vigilance, } \\
\text { Finger Tapping, TMT-A, TMT -B, } \\
\text { Digit Symbol }\end{array}$ & $\begin{array}{l}\text { Attrition: } 5 \% \\
\text { ITT: } N \\
\text { Blinding: } N\end{array}$ & $\begin{array}{l}\mathrm{AT}=.075 \\
\mathrm{EX}=.325\end{array}$ \\
\hline Fabre, 2002 (112) & $\begin{array}{l}32, \text { healthy elderly } \\
\text { adults, ages } 60 \text { to } \\
76\end{array}$ & $\begin{array}{l}\text { Duration: } 8 \text { wks } \\
\text { Frequency: } 45 \text { min, 2/wk } \\
\text { Intensity: - - - } \\
\text { Combined Strength } \\
\text { Training: } \mathrm{N} \\
\mathrm{MCl}: \mathrm{N}\end{array}$ & Wechsler Memory Scale & $\begin{array}{l}\text { Attrition: } 0 \% \\
\text { ITT: N } \\
\text { Blinding: N }\end{array}$ & $\begin{array}{l}\mathrm{EX}=-.188 \\
\mathrm{WM}=.878 \ddagger \\
\mathrm{ME}=-.339\end{array}$ \\
\hline Hassmen, 1992 (39) & $\begin{array}{l}\text { 32, all women, ages } \\
55 \text { to } 75\end{array}$ & $\begin{array}{l}\text { Duration: } 12 \text { wks } \\
\text { Frequency: } 20 \mathrm{~min}, 3 / \mathrm{wk} \\
\text { Intensity: } 9-13 \mathrm{RPE} \\
\text { Combined Strength } \\
\text { Training: N } \\
\mathrm{MCl}: \mathrm{N}\end{array}$ & $\begin{array}{l}\text { Digit Span, Face Recognition, } \\
\text { Simple Reaction Time, Choice } \\
\text { Reaction Time }\end{array}$ & $\begin{array}{l}\text { Attrition: } 7 \% \\
\text { ITT: N } \\
\text { Blinding: } N\end{array}$ & $\begin{array}{l}\mathrm{AT}=.179 \\
\mathrm{EX}=.167 \\
\mathrm{WM}=.204 \\
\mathrm{ME}=-.145\end{array}$ \\
\hline Hawkins, 1992 (66) & $\begin{array}{l}40, \text { sedentary, ages } 63 \\
\text { to } 82\end{array}$ & $\begin{array}{l}\text { Duration: } 10 \text { wks } \\
\text { Frequency: } 45 \text { min, 3/wk } \\
\text { Intensity: - - - } \\
\text { Combined Strength } \\
\text { Training: } \mathrm{N} \\
\mathrm{MCl}: \mathrm{N}\end{array}$ & $\begin{array}{l}\text { Single-Task Reaction Time, Dual- } \\
\text { Task Reaction Time, Difference } \\
\text { Between Single-Task And Dual- } \\
\text { Task Reaction Time }\end{array}$ & $\begin{array}{l}\text { Attrition: } 10 \% \\
\text { ITT: } \mathrm{N} \\
\text { Blinding: } \mathrm{N}\end{array}$ & $\begin{array}{l}\mathrm{AT}=-.243 \\
\mathrm{EX}=.047\end{array}$ \\
\hline Hoffman, 2008 (31) & $\begin{array}{l}\text { 153, sedentary and } \\
\text { depressed, ages } 41 \\
\text { to } 87\end{array}$ & $\begin{array}{l}\text { Duration: } 16 \text { wks } \\
\text { Frequency: } 45 \mathrm{~min}, 3 / \mathrm{wk} \\
\text { Intensity: } 70-85 \% \text { HRR } \\
\text { Combined Strength } \\
\text { Training: N } \\
\mathrm{MCl}: \mathrm{N}\end{array}$ & $\begin{array}{l}\text { Logical Memory, Verbal Paired } \\
\text { Associates, Digit Span, Animal } \\
\text { Naming, COWAT, Stroop Color } \\
\text { Word, Ruff } 2 \text { \& } 7 \text { Test, Digit } \\
\text { Symbol, TMT B-A }\end{array}$ & $\begin{array}{l}\text { Attrition: } 28 \% \\
\text { ITT: } Y \\
\text { Blinding: } Y\end{array}$ & $\begin{array}{l}\mathrm{AT}=.277 \\
\mathrm{EX}=.172 \\
\mathrm{WM}=-.031 \\
\mathrm{ME}=.072\end{array}$ \\
\hline Khatri, 2001 (113) & $\begin{array}{l}\text { 84, sedentary and } \\
\text { depressed, ages } 50 \\
\text { to } 72\end{array}$ & $\begin{array}{l}\text { Duration: } 17 \text { wks } \\
\text { Frequency: } 45 \text { min, 3/wk } \\
\text { Intensity: } 70-85 \% \text { HRR } \\
\text { Combined Strength } \\
\text { Training: N } \\
\mathrm{MCl}: \mathrm{N}\end{array}$ & $\begin{array}{l}\text { Visual Reproduction, Stroop Color- } \\
\text { Work Interference, Digit Span, } \\
\text { TMT-A, Digit Symbol, Stroop } \\
\text { Color, Stroop Word, TMT-B, } \\
\text { Logical Memory }\end{array}$ & $\begin{array}{l}\text { Attrition: } 25 \% \\
\text { ITT: } Y \\
\text { Blinding: } Y\end{array}$ & $\begin{array}{l}\mathrm{AT}=.121 \\
\mathrm{EX}=.291 \\
\mathrm{WM}=-.047 \\
\mathrm{ME}=.186\end{array}$ \\
\hline $\begin{array}{r}\text { Kramer, } 1999 \& \\
2002(74,75)\end{array}$ & $\begin{array}{l}124, \text { sedentary, ages } \\
60 \text { to } 75\end{array}$ & $\begin{array}{l}\text { Duration: } 26 \text { wks } \\
\text { Frequency: } 40 \mathrm{~min}, 3 / \mathrm{wk} \\
\text { Intensity: } 50-70 \% \text { HRR } \\
\text { Combined Strength } \\
\text { Training: N } \\
\mathrm{MCl}: \mathrm{N}\end{array}$ & $\begin{array}{l}\text { Reaction Time Tests: Switching } \\
\text { Trials, Non-Switching Trials, } \\
\text { Incompatible Trials, Compatible } \\
\text { Trials, Interference Effect } \\
\text { (Difference Between Compatible } \\
\text { Trials and Incompatible Trials), } \\
\text { Stop Signal Trials, Simple } \\
\text { Reaction-Time Trials }\end{array}$ & $\begin{array}{l}\text { Attrition: } 29 \% \\
\text { ITT:N } \\
\text { Blinding: } N\end{array}$ & $\begin{array}{l}\mathrm{AT}=.091 \\
\mathrm{EX}=.196 \\
\mathrm{WM}=-.101 \\
\mathrm{ME}=.156\end{array}$ \\
\hline
\end{tabular}


TABLE 1. Continued

\begin{tabular}{|c|c|c|c|c|c|}
\hline Author/Year & Sample & Intervention & Instruments & $\begin{array}{l}\text { Methodological } \\
\text { Characteristics }\end{array}$ & Hedge's G \\
\hline $\begin{array}{l}\text { Lautenschlager, } \\
2008 \text { (30) }\end{array}$ & $\begin{array}{l}\text { 170, elderly adults } \\
\text { with } \mathrm{MCl} \text {, age } M= \\
69\end{array}$ & $\begin{array}{l}\text { Duration: } 72 \mathrm{wks}^{*} \\
\text { Frequency: } 50 \mathrm{~min}, 3 / \mathrm{wk} \\
\text { Intensity: - - - } \\
\text { Combined Strength } \\
\text { Training: } \mathrm{N} \\
\mathrm{MCl}: \mathrm{Y}\end{array}$ & $\begin{array}{l}\text { Word list recall (immediate and } \\
\text { delayed), Digit Symbol, COWAT }\end{array}$ & $\begin{array}{l}\text { Attrition: } 19 \% \\
\text { ITT: } Y \\
\text { Blinding: } Y\end{array}$ & $\begin{array}{l}\mathrm{AT}=.083 \\
\mathrm{EX}=-.071 \\
\mathrm{ME}=.322^{\star *}\end{array}$ \\
\hline Masley, 2008 (114) & $\begin{array}{l}\text { 56, adults, age } M= \\
45\end{array}$ & $\begin{array}{l}\text { Duration: } 10 \text { wks } \\
\text { Frequency: } 5 / \mathrm{wk} \\
\text { Intensity: } 70-85 \% \mathrm{MHR} \\
\text { Combined Strength } \\
\text { Training: } \mathrm{Y} \\
\mathrm{MCl}: \mathrm{N}\end{array}$ & $\begin{array}{l}\text { CNS Vital Signs (verbal memory, } \\
\text { symbol digit coding, the Stroop } \\
\text { test, shifting attention, } \\
\text { continuous performance) }\end{array}$ & $\begin{array}{l}\text { Attrition: } 16 \% \\
\text { ITT: } \mathrm{N} \\
\text { Blinding: } \mathrm{N} \\
\text { (computerized) }\end{array}$ & $\begin{array}{l}\mathrm{AT}=-.158 \\
\mathrm{EX}=.487 \ddagger\end{array}$ \\
\hline Moul, 1995 (115) & $\begin{array}{l}30 \text {, sedentary, ages } 65 \\
\text { to } 72\end{array}$ & $\begin{array}{l}\text { Duration: } 8 \text { wks } \\
\text { Frequency: } 35 \text { min, 5/wk } \\
\text { Intensity: } 60-65 \% \text { HRR } \\
\text { Combined Strength } \\
\text { Training: N } \\
\mathrm{MCl}: \mathrm{N}\end{array}$ & $\begin{array}{l}\text { Ross Information Processing } \\
\text { Assessment Subtests: } \\
\text { Organization, Auditory } \\
\text { Processing, Immediate Memory, } \\
\text { Recent Memory, Temporal } \\
\text { Orientation, Problem Solving/ } \\
\text { Abstract Reasoning }\end{array}$ & $\begin{array}{l}\text { Attrition: } 0 \% \\
\text { ITT: N } \\
\text { Blinding: } N\end{array}$ & $\begin{array}{l}\mathrm{EX}=.780 \ddagger \\
\mathrm{ME}=.351\end{array}$ \\
\hline $\begin{array}{l}\text { Munguia-Izquierdo, } \\
2008 \text { (116) }\end{array}$ & $\begin{array}{l}\text { 60, middle-aged } \\
\text { women with } \\
\text { fibromyalgia, ages } \\
18 \text { to } 60\end{array}$ & $\begin{array}{l}\text { Duration: } 16 \text { wks } \\
\text { Frequency: } 50 \mathrm{~min}, 3 / \mathrm{wk} \\
\text { Intensity: } 50-80 \% \mathrm{MHR} \\
\text { Combined Strength } \\
\text { Training: } \mathrm{N} \\
\mathrm{MCl}: \mathrm{N}\end{array}$ & $\begin{array}{l}\text { Paced Auditory Serial Addition Task } \\
\text { (PASAT) }\end{array}$ & $\begin{array}{l}\text { Attrition: } 12 \% \\
\text { ITT: } Y \\
\text { Blinding: } Y\end{array}$ & AT $=.922^{* \star *}$ \\
\hline Oken, 2004 (117) & $\begin{array}{l}\text { 69, multiple sclerosis, } \\
\qquad M=49\end{array}$ & $\begin{array}{l}\text { Duration: } 26 \text { wks } \\
\text { Frequency: } 90 \text { min, 1/wk } \\
\text { Intensity: - - - } \\
\text { Combined Strength } \\
\text { Training: } \mathrm{N} \\
\mathrm{MCl}: \mathrm{N}\end{array}$ & $\begin{array}{l}\text { Stroop Color-Word test, Simple } \\
\text { Reaction Time, Complex Reaction } \\
\text { Time, Attentional Shift Task, } \\
\text { PASAT, Logical Memory, WAIS } \\
\text { Similarities }\end{array}$ & $\begin{array}{l}\text { Attrition }=12 \% \\
\text { ITT: } N \\
\text { Blinding: } \mathrm{Y}\end{array}$ & $\begin{array}{l}\mathrm{AT}=.074 \\
\mathrm{EX}=.133 \\
\mathrm{WM}=-.354 \\
\mathrm{ME}=.000\end{array}$ \\
\hline Oken, 2006 (118) & $\begin{array}{l}135, \text { healthy adults, } \\
\text { ages } 65 \text { to } 85\end{array}$ & $\begin{array}{l}\text { Duration: } 26 \text { wks } \\
\text { Frequency: } 60 \mathrm{~min}, 1 / \mathrm{wk} \\
\text { Intensity: } 70 \% \text { HRR } \\
\text { Combined Strength } \\
\text { Training: } \mathrm{N} \\
\mathrm{MCl}: \mathrm{N}\end{array}$ & $\begin{array}{l}\text { Stroop Interference, Word List } \\
\text { Recall, Letter-Number } \\
\text { Sequencing, Covert Orienting, } \\
\text { Divided Attention, Set Shifting, } \\
\text { Simple Reaction time, Complex } \\
\text { Reaction time }\end{array}$ & $\begin{array}{l}\text { Attrition: } 13 \% \\
\text { ITT: N } \\
\text { Blinding: } Y\end{array}$ & $\begin{array}{l}\mathrm{AT}=-.132 \\
\mathrm{EX}=-.034 \\
\mathrm{WM}=-.029 \\
\mathrm{ME}=-.055\end{array}$ \\
\hline Okumiya, 1996 (29) & $\begin{array}{l}\text { 42, healthy older } \\
\text { adults, ages } 75 \text { to } \\
87\end{array}$ & $\begin{array}{l}\text { Duration: } 24 \text { wks } \\
\text { Frequency: } 60 \mathrm{~min}, 3 / \mathrm{wk} \\
\text { Intensity: - - - } \\
\text { Combined Strength } \\
\text { Training: } \mathrm{Y} \\
\mathrm{MCl}: \mathrm{N}\end{array}$ & $\begin{array}{l}\text { MMSE, Hasegawa Dementia Scale, } \\
\text { Visuospatial Cognitive } \\
\text { Performance Test }\end{array}$ & $\begin{array}{l}\text { Attrition: } 0 \% \\
\text { ITT: N } \\
\text { Blinding: } N\end{array}$ & $\mathrm{AT}=.938^{\star *}$ \\
\hline Panton, 1990 (119) & $\begin{array}{l}\text { 39, healthy untrained } \\
\text { older adults, ages } 70 \\
\text { to } 79\end{array}$ & $\begin{array}{l}\text { Duration: } 26 \text { wks } \\
\text { Frequency: } 45 \text { min, 3/wk } \\
\text { Intensity: } 75 \% \text { HRR } \\
\text { Combined Strength } \\
\text { Training: } \mathrm{N} \\
\mathrm{MCl}: \mathrm{N}\end{array}$ & $\begin{array}{l}\text { Reaction time, Speed of Movement } \\
\text { Time }\end{array}$ & $\begin{array}{l}\text { Attrition: } 14 \% \\
\text { ITT: N } \\
\text { Blinding: N }\end{array}$ & $\mathrm{AT}=.111$ \\
\hline Perri, 1984 (121) & $\begin{array}{l}42 \text {, healthy older } \\
\text { adults, ages } 60 \text { to } \\
79\end{array}$ & $\begin{array}{l}\text { Duration: } 15 \text { wks } \\
\text { Frequency: } 30 \mathrm{~min}, 3 / \mathrm{wk} \\
\text { Intensity: } 40-50 \% \mathrm{HRR} \\
\text { Combined Strength } \\
\text { Training: } \mathrm{N} \\
\mathrm{MCl}: \mathrm{N}\end{array}$ & Rey Auditory Verbal Learning Task & $\begin{array}{l}\text { Attrition: } 41 \% \\
\text { ITT: N } \\
\text { Blinding: N }\end{array}$ & $\mathrm{ME}=.261$ \\
\hline Pierce, 1993 (120) & $\begin{array}{l}\text { 90, middle-aged adults } \\
\text { with hypertension, } \\
\text { ages 29-59 }\end{array}$ & $\begin{array}{l}\text { Duration: } 16 \text { wks } \\
\text { Frequency: } 50 \mathrm{~min}, 3 / \mathrm{wk} \\
\text { Intensity: } 70 \% \text { HRR } \\
\text { Combined Strength } \\
\text { Training: } \mathrm{N} \\
\mathrm{MCl}: \mathrm{N}\end{array}$ & $\begin{array}{l}\text { Digit Symbol, Stroop Color Word } \\
\text { test, Digit Span, TMT-B, } \\
\text { Sternberg Memory Search Task } \\
\text { (Slope and Y-intercept), Verbal } \\
\text { Paired Associates, Logical Memory } \\
\text { (immediate and delayed), Figural } \\
\text { Memory (immediate and delayed) }\end{array}$ & $\begin{array}{l}\text { Attrition: } 7 \% \\
\text { ITT: } Y \\
\text { Blinding: } Y\end{array}$ & $\begin{array}{l}\mathrm{AT}=.249 \\
\mathrm{EX}=.126 \\
\mathrm{WM}=-.283 \\
\mathrm{ME}=.233\end{array}$ \\
\hline
\end{tabular}


AEROBIC EXERCISE AND NEUROCOGNITION

TABLE 1. Continued

\begin{tabular}{|c|c|c|c|c|c|}
\hline Author/Year & Sample & Intervention & Instruments & $\begin{array}{l}\text { Methodological } \\
\text { Characteristics }\end{array}$ & Hedge's G \\
\hline Russell, 1982 (122) & $\begin{array}{l}\text { 45, sedentary older } \\
\text { adults, ages } 55 \text { to } \\
70\end{array}$ & $\begin{array}{l}\text { Duration: } 16 \text { wks } \\
\text { Frequency: } 45 \mathrm{~min}, 3 / \mathrm{wk} \\
\text { Intensity: - - - } \\
\text { Combined Strength } \\
\text { Training: N } \\
\mathrm{MCl}: \mathrm{N}\end{array}$ & $\begin{array}{l}\text { Simple Reaction Time, Complex } \\
\text { Reaction Time }\end{array}$ & $\begin{array}{l}\text { Attrition: } 4 \% \\
\text { ITT: } N \\
\text { Blinding: } N\end{array}$ & $\begin{array}{l}\mathrm{AT}=.214 \\
\mathrm{EX}=.081\end{array}$ \\
\hline Scherder, 2005 (79) & $\begin{array}{l}\text { 43, elderly adults with } \\
\mathrm{MCl} \text {, ages } 76 \text { to } 94\end{array}$ & $\begin{array}{l}\text { Duration: } 6 \text { wks } \\
\text { Frequency: } 30 \mathrm{~min}, 3 / \mathrm{wk} \\
\text { Intensity: - - - } \\
\text { Combined Strength } \\
\text { Training: } \mathrm{N} \\
\mathrm{MCl}: \mathrm{Y}\end{array}$ & $\begin{array}{l}\text { Category Naming, TMT-A, TMT-B, } \\
\text { Digit Span, Visual Memory Span, } \\
\text { Rivermead Behavioral Memory } \\
\text { Test (Faces and Pictures), Verbal } \\
\text { Learning and Memory Test: } \\
\text { Direct Recall, Delayed Recall, and } \\
\text { Recognition }\end{array}$ & $\begin{array}{l}\text { Attrition: } 7 \% \\
\text { ITT: N } \\
\text { Blinding: Y }\end{array}$ & $\begin{array}{l}\mathrm{EX}=.441 \\
\mathrm{WM}=.037 \\
\mathrm{ME}=.413\end{array}$ \\
\hline Smiley-Oyen, 2008 (123) & $\begin{array}{l}\text { 57, older adults, ages } \\
65-79\end{array}$ & $\begin{array}{l}\text { Duration: } 40 \text { wks } \\
\text { Frequency: } 25-30 \mathrm{~min}, 3 / \mathrm{wk} \\
\text { Intensity: } 65-80 \% \text { HRR } \\
\text { Combined Strength } \\
\text { Training: N } \\
\text { MCl: N }\end{array}$ & $\begin{array}{l}\text { Stroop Test, Go-No-Go Test, Simple } \\
\text { Reaction Time, Choice Reaction } \\
\text { Time, Wisconsin Card Sorting } \\
\text { Test }\end{array}$ & $\begin{array}{l}\text { Attrition: } 7 \% \\
\text { ITT: N } \\
\text { Blinding: } N\end{array}$ & $\begin{array}{l}\mathrm{AT}=.234 \\
\mathrm{EX}=-.092\end{array}$ \\
\hline Stroth, 2009 (124) & $\begin{array}{l}\text { 28, young adults, age } \\
\quad M=20\end{array}$ & $\begin{array}{l}\text { Duration: } 6 \mathrm{wks} \\
\text { Frequency: } 30 \mathrm{~min}, 3 / \mathrm{wk} \\
\text { Intensity: } 70-100 \% \text { aerobic } \\
\text { threshold } \\
\text { Combined Strength } \\
\text { Training: } \mathrm{N} \\
\mathrm{MCl}: \mathrm{N}\end{array}$ & $\begin{array}{l}\text { Digit Symbol Substitution Test, Rey } \\
\text { Auditory Verbal Learning Test, } \\
\text { Stroop Test }\end{array}$ & $\begin{array}{l}\text { Attrition: } 22 \% \\
\text { ITT: } N \\
\text { Blinding: } Y\end{array}$ & $\begin{array}{l}\mathrm{AT}=-.123 \\
\mathrm{ME}=.650 \ddagger\end{array}$ \\
\hline Wallman, 2004 (125) & $\begin{array}{l}\text { 61, adults with chronic } \\
\text { fatigue syndrome, } \\
\text { ages } 16 \text { to } 74\end{array}$ & $\begin{array}{l}\text { Duration: } 12 \text { wks } \\
\text { Frequency: increased } \\
\text { progressively Intensity: based } \\
\text { on target HR } \\
\text { from treadmill testing } \\
\text { Combined Strength } \\
\text { Training: N } \\
\mathrm{MCl}: \mathrm{N}\end{array}$ & $\begin{array}{l}\text { Stroop Test (82 questions) Stroop } \\
\text { Test (95 questions) }\end{array}$ & $\begin{array}{l}\text { Attrition: } 10 \% \\
\text { ITT: } N \\
\text { Blinding: } Y\end{array}$ & $\mathrm{EX}=.479 *$ \\
\hline Whitehurst, 1991 (126) & $\begin{array}{l}\text { 14, sedentary older } \\
\text { women, ages } 61 \text { to } \\
73\end{array}$ & $\begin{array}{l}\text { Duration: } 8 \text { wks } \\
\text { Frequency: } 35 \text { min, 3/wk } \\
\text { Intensity: - - - } \\
\text { Combined Strength } \\
\text { Training: N } \\
\text { MCl: N }\end{array}$ & $\begin{array}{l}\text { Simple Reaction Time, Choice } \\
\text { Reaction Time }\end{array}$ & $\begin{array}{l}\text { Attrition: } 0 \% \\
\text { ITT: } N \\
\text { Blinding: } N\end{array}$ & $\begin{array}{l}\mathrm{AT}=-.551 \\
\mathrm{EX}=-.609\end{array}$ \\
\hline Williams, 1997 (104) & $\begin{array}{l}\text { 187, all women, age } M \\
\quad=72\end{array}$ & $\begin{array}{l}\text { Duration: } 42 \text { wks } \\
\text { Frequency: } 35 \text { min, 2/wk } \\
\text { Intensity: - - - } \\
\text { Combined Strength } \\
\text { Training: Y } \\
\mathrm{MCl}: \mathrm{N}\end{array}$ & $\begin{array}{l}\text { Digit Span, Picture Arrangement, } \\
\text { Cattell's Matrices }\end{array}$ & $\begin{array}{l}\text { Attrition: } 20 \% \\
\text { ITT: N } \\
\text { Blinding: N }\end{array}$ & $\begin{array}{l}\mathrm{AT}=.501^{* *} \\
\mathrm{EX}=.189 \\
\mathrm{WM}=.348^{*}\end{array}$ \\
\hline Williamson, 2009 (34) & $\begin{array}{l}102, \text { elderly adults, } \\
\text { ages } 70-89 \text { years }\end{array}$ & $\begin{array}{l}\text { Duration: } 52 \text { wks } \\
\text { Frequency: } 45 \text { min, 1-2/wk } \\
\text { Intensity: - - - } \\
\text { Combined Strength } \\
\text { Training: Y } \\
\mathrm{MCl}: \mathrm{N}\end{array}$ & $\begin{array}{l}\text { Digit Symbol, Modified Stroop Test, } \\
\text { 3MSE, Rey Auditory Verbal } \\
\text { Learning Test }\end{array}$ & $\begin{array}{l}\text { Attrition: } 10 \% \\
\text { ITT: } N \\
\text { Blinding: } \mathrm{Y}\end{array}$ & $\begin{array}{l}\mathrm{AT}=.206 \\
\mathrm{EX}=.026 \\
\mathrm{ME}=.011\end{array}$ \\
\hline van Uffelen, 2008 (33) & $\begin{array}{l}\text { 152, elderly adults } \\
\text { with } \mathrm{MCl} \text {, age } M= \\
75\end{array}$ & $\begin{array}{l}\text { Duration: } 52 \text { wks } \\
\text { Frequency: } 60 \text { min, 2/wk } \\
\text { Intensity: }>3 \text { METs } \\
\text { Combined Strength } \\
\text { Training: } \mathrm{N} \\
\mathrm{MCl}: \mathrm{Y}\end{array}$ & $\begin{array}{l}\text { Digit Symbol, Stroop Color Word } \\
\text { Test,Verbal Fluency, Auditory } \\
\text { Verbal Learning Test }\end{array}$ & $\begin{array}{l}\text { Attrition: } 9 \% \\
\text { ITT: } Y \\
\text { Blinding: } Y\end{array}$ & $\begin{array}{l}\mathrm{AT}=-.10 \\
\mathrm{EX}=-.04 \\
\mathrm{ME}=-.03\end{array}$ \\
\hline
\end{tabular}

*** $\mathrm{p}<.001 ; * * \mathrm{p}<.01 ; * \mathrm{p}<.05 ; \ddagger \mathrm{p}<.10 ; \mathrm{AT}=$ attention and processing speed; EX = executive function; MET = metabolic equivalent, WM = working memory; $\mathrm{MCI}=$ Mild Cognitive Impairment, $\mathrm{ME}=$ memory; HRR $=$ Heart Rate Reserve; MHR = maximum heart rate; RPE $=$ Ratings of Perceived Exertion; TMT $=$ Trail Mating Test; $¥=$ indicates multiple time points of data. 
TABLE 2. Effects of Aerobic Exercise Interventions Versus Controls on Neurocognitive Performance for Various Cognitive Indices

\begin{tabular}{|c|c|c|c|c|}
\hline Cognitive Test & Studies & Domain & Hedge's G $(95 \% \mathrm{Cl})$ (Random Effects) & $p$ \\
\hline Digit Symbol Substitution & 8 & Attention/Processing Speed & $0.146(-0.002$ to 0.294$)$ & .052 \\
\hline Complex/Choice Reaction Time & 8 & Attention/Processing Speed & $0.112(-0.064$ to 0.288$)$ & .898 \\
\hline Simple Reaction Time & 8 & Attention/Processing Speed & $0.088(-0.118$ to 0.295$)$ & .116 \\
\hline Ruff 2 and 7 Test & 2 & Attention/Processing Speed & $0.052(-0.224$ to 0.327$)$ & .715 \\
\hline Trail Making Test Section A & 2 & Attention/Processing Speed & $0.169(-0.144$ to 0.482$)$ & .291 \\
\hline Stroop Interference & 7 & Executive Function & $0.027(-0.149$ to 0.204$)$ & .761 \\
\hline Trail Making Test Section B & 5 & Executive Function & $0.234(0.042$ to 0.426$)$ & .017 \\
\hline Animal Naming & 4 & Executive Function & $0.275(0.006$ to 0.545$)$ & .045 \\
\hline COWAT & 2 & Executive Function & $-0.015(-0.239$ to 0.229$)$ & .894 \\
\hline Logical Memory, Immediate Recall & 5 & Memory & $0.151(-0.050$ to 352$)$ & .140 \\
\hline Rey Auditory Verbal Learning Test & 4 & Memory & $0.113(-0.082$ to 0.308$)$ & .255 \\
\hline Digit Span & 6 & Working Memory & $0.065(-0.079$ to 0.209$)$ & .373 \\
\hline WAIS Letter-Number Sequencing & 2 & Working Memory & $-0.134(-0.469$ to 0.202$)$ & .435 \\
\hline
\end{tabular}

$\mathrm{CI}=$ confidence interval; COWAT $=$ Controlled Oral Word Association Test; WAIS $=$ Wechsler Adult Intelligence Scale.

TABLE 3. Classification of Neurocognitive Tests by Domain

Neurocognitive Domain

\begin{tabular}{|c|c|c|c|}
\hline Attention & Executive Function & Working Memory & Memory \\
\hline $\begin{array}{l}\text { Accuracy Index } \\
\text { Complex/Choice RT } \\
\text { d2 Test of Attention } \\
\text { Digit Matching RTe } \\
\text { Digit Symbol Substitution Test } \\
\text { Mental Speed } \\
\text { Paced Auditory Serial Attention } \\
\text { Test (PASAT) } \\
\text { Picture Arrangement } \\
\text { Premotor Time } \\
\text { Response Compatibility RT } \\
\text { Ruff } 2 \text { and } 7 \text { Test (Letters) } \\
\text { Simple RT } \\
\text { Single/Choice Time Sharing } \\
\text { Spatial Attention Task } \\
\text { Speed of Movement } \\
\text { Stopping Task RT } \\
\text { Stroop Color } \\
\text { Stroop Word } \\
\text { Task Switching RT } \\
\text { Trail Making Test Part A } \\
\text { Visuospatial Cognitive Performance } \\
\text { Test } \\
\text { Word Copying Speed }\end{array}$ & $\begin{array}{l}\text { Attentional Flexibility } \\
\text { Categorical Fluency (Animal Naming) } \\
\text { Cattell's Matrices } \\
\text { Cognitive Flexibility } \\
\text { Covert Orienting of Attention Task } \\
\text { Go-No-Go Test } \\
\text { Mental Control } \\
\text { Nonverbal Fluency Test } \\
\text { Number Copying Speed } \\
\text { RIPA Organization } \\
\text { RIPA Problem Solving } \\
\text { RIPA Abstract Reasoning } \\
\text { Ruff } 2 \text { and } 7 \text { Test (Digits) } \\
\text { Selective Reminding Intrusions } \\
\text { Set Shifting Ability } \\
\text { Stopping Task } \\
\text { Stroop Color/Word or Interference } \\
\text { Trail Making Test Part B } \\
\text { Useful Field of View } \\
\text { Verbal Fluency Test (FAS) } \\
\text { WAIS Similarities } \\
\text { Wisconsin Card Sorting Task }\end{array}$ & $\begin{array}{l}\text { Digit Span } \\
\text { N-Back Spatial Task } \\
\text { N-Back Task } \\
\text { Self-Ordered Pointing } \\
\text { Visual Memory span } \\
\text { WAIS Letter Number } \\
\text { Sequencing }\end{array}$ & $\begin{array}{l}\text { ADAS Word List Recall } \\
\text { Auditory Verbal Learning Test } \\
\text { Benton Visual Retention Test } \\
\text { CERAD delayed recall } \\
\text { RAVLT } \\
\text { RAVLT Delay } \\
\text { RAVLT, Temporal Order } \\
\text { RBMT faces } \\
\text { RBMT pictures } \\
\text { RIPA Auditory Processing } \\
\text { RIPA Immediate Memory } \\
\text { RIPA Recent Memory } \\
\text { Sternberg Memory Search Task, } \\
\text { Y-intercept } \\
\text { Sternberg Memory Search Task, Slope } \\
\text { Visual and Verbal Memory Test } \\
\text { Visual Reproduction, Immediate } \\
\text { Visual Reproductions } \\
\text { VLMT Delayed Recall } \\
\text { VLMT Direct Recall } \\
\text { VLMT Recognition } \\
\text { WMS Facial Recognition } \\
\text { WMS Figural Memory, Immediate } \\
\text { WMS Figural Memory, Delayed } \\
\text { WMS Logical Memory, Immediate } \\
\text { WMS Logical Memory, Delayed } \\
\text { WMS Verbal Paired Associates } \\
\text { WMS Visual Reproduction }\end{array}$ \\
\hline
\end{tabular}

ADAS = Alzheimer's Disease Assessment Scale; RT = reaction time; CERAD = Consortium to Establish a Registry for Alzheimer's Disease; RAVLT $=$ Rey Auditory Verbal Learning Task; WAIS $=$ Wechsler Adult Intelligence Scale; PASAT $=$ Paced Auditory Serial Attention Test; RBMT $=$ Rivermead Behavioral Memory Test; RIPA = Ross Information Processing Test; VLMT = Verbal Learning and Memory Test; WMS = Wechsler Memory Scale.

stretching and toning, health education, and relaxation exercises were also used. Rates of attrition varied widely (range, $0 \%-41 \%$; mean attrition, $12.2 \%)$. Only $13(44.8 \%)$ studies utilized blinded assessments and only seven (24.1\%) studies utilized ITT analyses. The effects of exercise on individual neurocognitive measures are presented in Table 2. Due to the substantial number and heterogeneity of neurocognitive tests (Table 3), only those tests used in more than one study are presented.

\section{Attention and Processing Speed}

Twenty-four studies examined the effects of aerobic exercise on attention and processing speed. Exercise training was 


\section{Attention and Processing Speed}

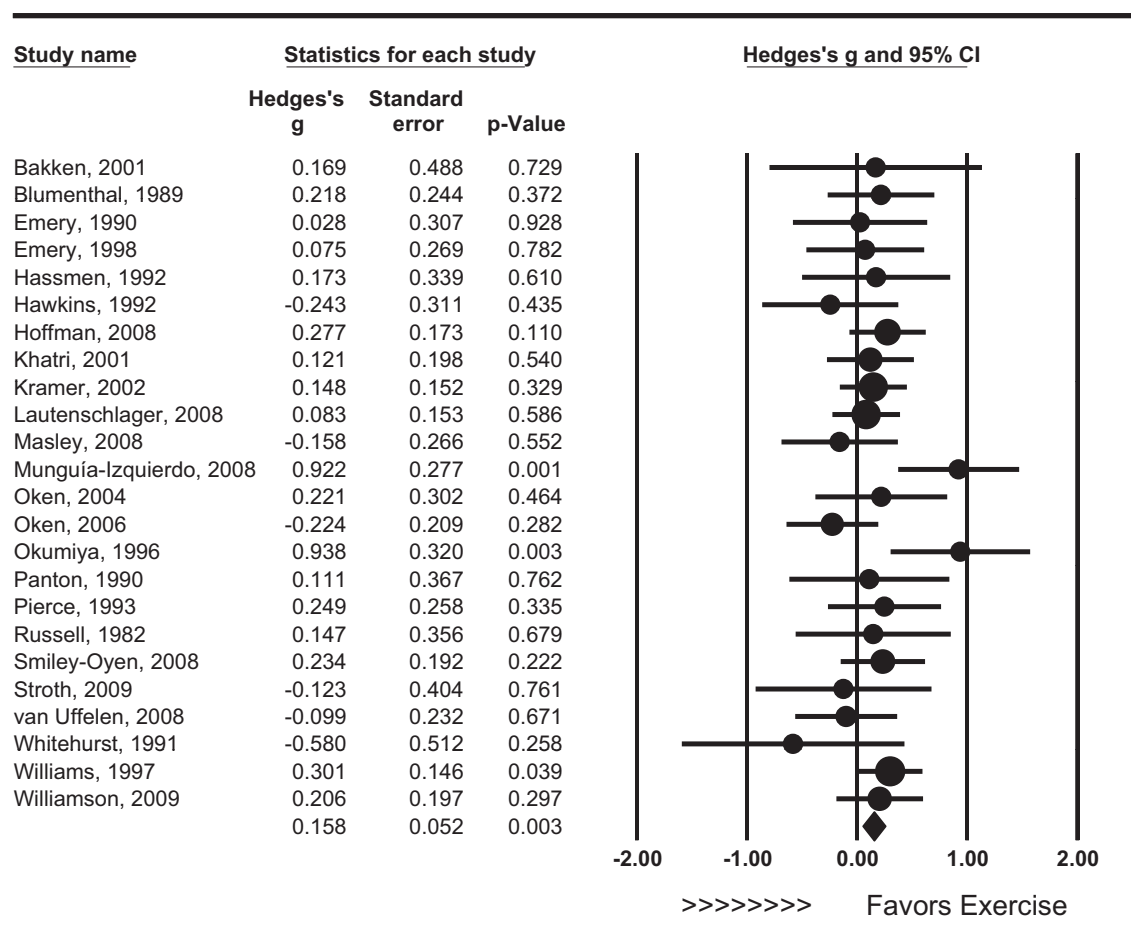

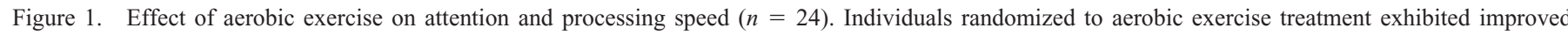

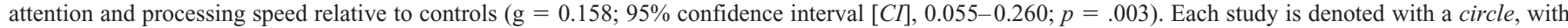
larger sample sizes corresponding to larger marks.

associated with modest improvements in attention and processing speed $(\mathrm{g}=0.158 ; 95 \%$ confidence interval $[\mathrm{CI}]$, 0.055-0.260; $p=.003$ ) (Fig. 1) and this effect was consistent across studies $\left(\mathrm{Q}_{23}=26.249, p=.289\right)$. Moderator analyses demonstrated that trials of greater duration did not improve attention and processing speed to a greater extent than briefer interventions $\left(r=.17, \mathrm{Q}_{1}=3.555, p=.399\right)$. Similarly, intensity was not associated with variations in attention and processing speed outcomes $\left(r=-.375, \mathrm{Q}_{1}=1.41, p=.235\right)$. Results did not differ between individuals with MCI ( $\mathrm{g}=$ $0.028, p<.001)$ and other samples $(\mathrm{g}=0.181, p=.825)$ $\left(\mathrm{Q}_{1}=1.228, p=.268\right)$. Combined interventions improved attention and processing speed to a greater extent $(\mathrm{g}=0.350$; $95 \% \mathrm{CI}, 0.042-0.658 ; p=.026)$ than aerobic only interventions $(\mathrm{g}=0.098 ; 95 \% \mathrm{CI},-0.012$ to $0.208 ; p=.152)\left(\mathrm{Q}_{1}=\right.$ $4.373, p=.037)$. There was no observed association between the mean age of study participants and improvements in attention and processing speed $(r=-.047, p=.817)$.

\section{Executive Function}

Nineteen studies assessed the effects of aerobic exercise on executive function. Aerobic exercise was associated with modest improvements in executive function $(\mathrm{g}=0.123 ; 95 \%$ CI, $0.021-0.225 ; p=.018$ ) (Fig. 2), and effects were of similar magnitude across studies $\left(\mathrm{Q}_{18}=13.418, p=.766\right)$. Neither duration $\left(r=-.436, \mathrm{Q}_{1}=3.627, p=.057\right)$ nor intensity $\left(r=-.203, \mathrm{Q}_{1}=0.413, p=.520\right)$ were related to improved executive function. Improvements in executive function were smaller among individuals with $\mathrm{MCI}(\mathrm{g}=$ $-0.004, p=.973$ ) relative to other samples $(\mathrm{g}=0.153, p=$ .008) $\left(\mathrm{Q}_{1}=1.377, p=.241\right)$, and findings did not differ between studies that included only aerobic exercise $(\mathrm{g}=$ $0.109, p=.074)$ or combined aerobic exercise with other exercises (e.g., strength training) $(\mathrm{g}=0.163, p=.106)\left(\mathrm{Q}_{1}=\right.$ $0.214, p=.644)$. Finally, there was no observed association between the mean age of study participants and improvements in executive function $(r=-.348, p=.130)$.

\section{Working Memory}

Twelve studies examined the effects of aerobic exercise on working memory. Exercise did not seem to improve working memory performance $(\mathrm{g}=0.032 ; 95 \% \mathrm{CI},-0.103$ to $0.166 ; p=$ .642) (Fig. 3) and this effect was relatively consistent across trials $\left(\mathrm{Q}_{11}=12.241, p=.346\right)$. Similar to other cognitive domains, neither the duration of the intervention $\left(r=.346, \mathrm{Q}_{1}=1.438\right.$, $p=.230)$ nor the intensity of exercise $\left(r=.109, \mathrm{Q}_{1}=0.123\right.$, $p=.725)$ seemed to moderate the effects of treatment. Only one study examined the effects of working memory among individuals with MCI and test for moderation was, therefore, not examined. Combined interventions $(n=2)$ seemed to improve working memory $\left(\mathrm{Q}_{1}=4.817, p=.028\right)(\mathrm{g}=0.288 ; 95 \% \mathrm{CI}$, $0.030-0.546 ; p=.028)$ relative to aerobic only interventions ( $\mathrm{g}=-0.042 ; 95 \% \mathrm{CI},-0.184$ to $0.101 ; p=.567)$. In 


\section{Executive Function}

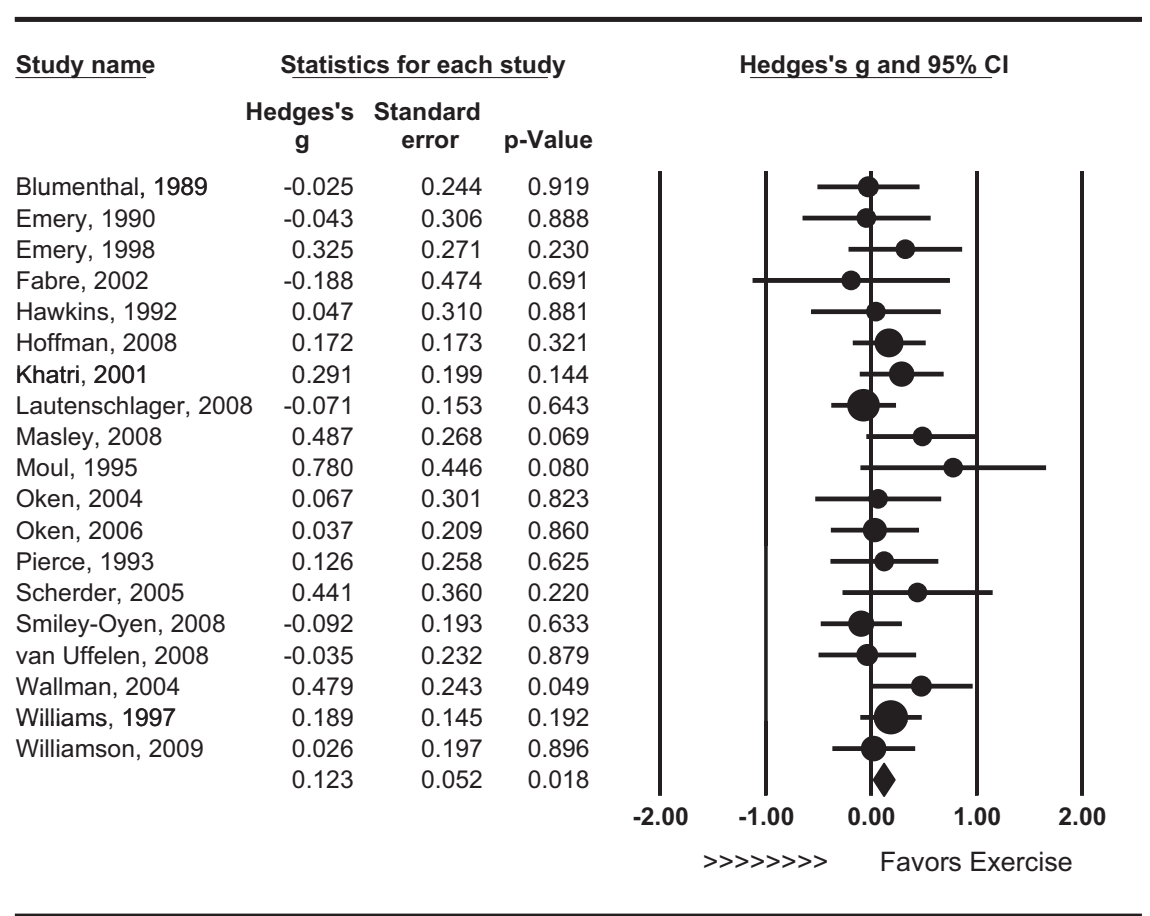

Figure 2. Effect of aerobic exercise on executive function $(n=19)$. Individuals randomized to aerobic exercise treatment exhibited improved executive function $(\mathrm{g}=0.123 ; 95 \%$ confidence interval $[C], 0.021-0.225 ; p=.018)$. Each study is denoted with a circle, with larger sample sizes corresponding to larger marks.

Working Memory

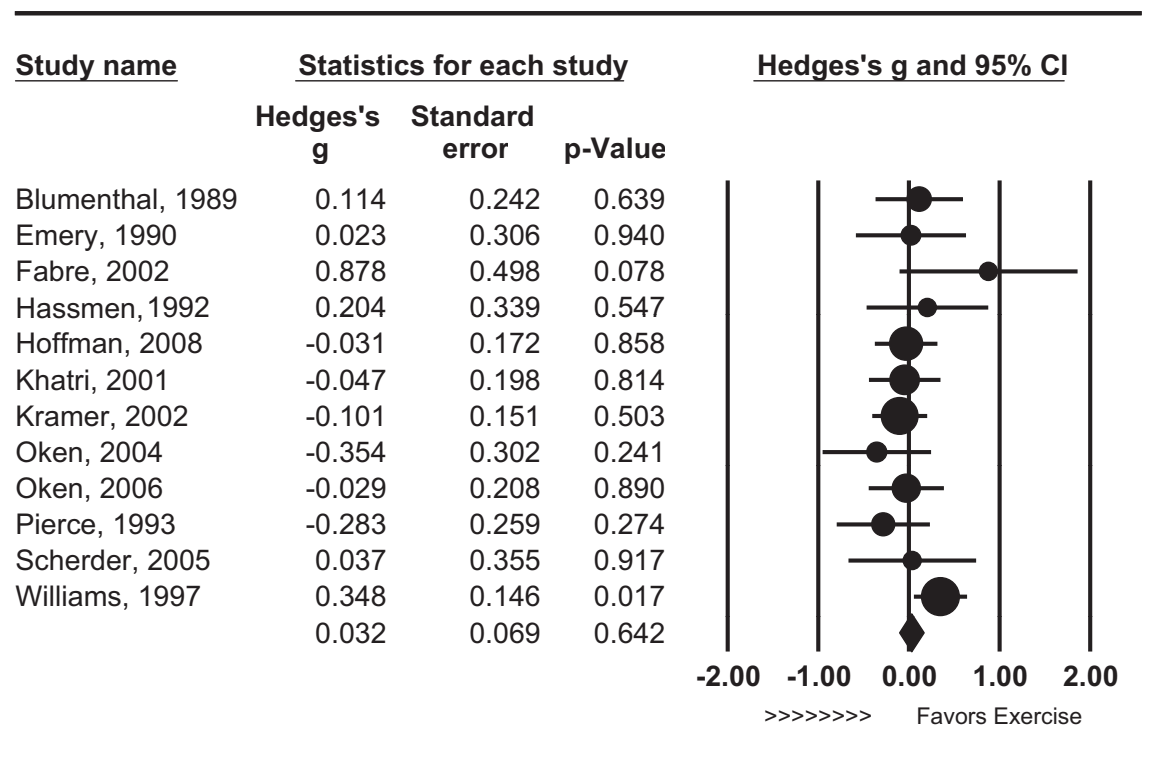

Figure 3. Effect of aerobic exercise on working memory $(n=12)$. Individuals randomized to aerobic exercise treatment did not exhibit significant improvements in working memory relative to controls ( $\mathrm{g}=0.032 ; 95 \%$ confidence interval $[C I],-0.103$ to $0.166 ; p=.642$ ). Each study is denoted with a circle, with larger sample sizes corresponding to larger marks.

addition, a significant association was observed between mean age of study participants and improvements in working memory, with older samples demonstrating greater improvements relative to younger samples $(r=.564, p=.051)$.

\section{Memory}

Sixteen studies assessed the effects of aerobic exercise on memory function. Aerobic exercise was associated with modest improvements in memory relative to controls $(g=0.128$; 


\section{Memory}

\begin{tabular}{|c|c|c|c|c|c|c|c|}
\hline \multirow[t]{2}{*}{ Study name } & \multicolumn{3}{|c|}{ Statistics for each study } & & \multicolumn{3}{|c|}{ Hedges's $\mathrm{g}$ and $95 \% \mathrm{Cl}$} \\
\hline & $\begin{array}{l}\text { Hedges's } \\
\text { g }\end{array}$ & $\begin{array}{l}\text { Standard } \\
\text { error }\end{array}$ & p-Value & & & & \\
\hline Blumenthal, 1989 & -0.066 & 0.242 & 0.786 & & & & \\
\hline Fabre, 2002 & -0.339 & 0.477 & 0.477 & & & & \\
\hline Hassmen, 1992 & -0.145 & 0.335 & 0.665 & & & & \\
\hline Hoffman, 2008 & 0.072 & 0.173 & 0.676 & & & & \\
\hline Khatri, 2001 & 0.186 & 0.198 & 0.348 & & & & \\
\hline Kramer, 2002 & 0.156 & 0.151 & 0.304 & & & & \\
\hline Lautenschlager, 2008 & 0.322 & 0.154 & 0.036 & & & & \\
\hline Moul, 1995 & 0.351 & 0.436 & 0.421 & & & & \\
\hline Oken, 2004 & 0.000 & 0.299 & 1.000 & & & & \\
\hline Oken, 2006 & -0.055 & 0.208 & 0.793 & & & & \\
\hline Perri, 1985 & 0.261 & 0.276 & 0.345 & & & & \\
\hline Pierce, 1993 & 0.233 & 0.259 & 0.369 & & & & \\
\hline Scherder, 2005 & 0.413 & 0.360 & 0.251 & & & & \\
\hline Stroth, 2009 & 0.650 & 0.378 & 0.085 & & & & \\
\hline van Uffelen, 2008 & -0.029 & 0.232 & 0.900 & & & & \\
\hline \multirow[t]{4}{*}{ Williamson, 2009} & 0.011 & 0.197 & 0.954 & & & & \\
\hline & 0.128 & 0.058 & 0.026 & & & & \\
\hline & & & & -2.00 & -1.00 & 1.00 & 2.00 \\
\hline & & & & & >>>>> & Favors Exer & \\
\hline
\end{tabular}

Figure 4. Effect of aerobic exercise on memory $(n=16)$. Individuals randomized to aerobic exercise treatment exhibited improved memory relative to controls $(\mathrm{g}=0.128 ; 95 \%$ confidence interval $[C I], 0.015-0.241 ; p=.026)$. Each study is denoted with a circle, with larger sample sizes corresponding to larger marks.

95\% CI, 0.015-0.241; $p=.026$ ) (Fig. 4) and effects were of similar magnitude across studies $\left(\mathrm{Q}_{15}=9.030, p=.876\right)$. Neither intensity $\left(r=-.051, \mathrm{Q}_{1}=0.026, p=.871\right)$ nor duration $\left(r=.373, \mathrm{Q}_{1}=1.381, p=.240\right)$ seemed to moderate the observed effects on memory. Sensitivity analyses demonstrated that the effects of exercise were stronger among individuals with $\mathrm{MCI}(\mathrm{g}=0.237 ; 95 \% \mathrm{CI}, 0.000-0.474 ; p=$ $.050)$ relative to noncognitively compromised individuals ( $\mathrm{g}=0.096 ; 95 \% \mathrm{CI},-0.032$ to $0.224 ; p=.143)$, although the statistical test for moderation did not achieve significance $\left(\mathrm{Q}_{1}=1.055, p=.304\right)$. Only one study assessing memory utilized a combined intervention, so this was not examined as a potential moderator. In addition, there was no observed association between the mean age of study participants and improvements in memory $(r=-.222, p=.175)$.

\section{Study Quality}

In order to assess whether methodological quality moderated the observed pattern of results, we examined whether treatment effects varied by 1) blinding of assessors and 2) use of ITT analyses. Studies did not differ in neurocognitive treatment effects whether they did ( $\mathrm{g}=0.143, p=.013)$ or did not use $(\mathrm{g}=0.185, p=.012)$ blinded assessments $\left(\mathrm{Q}_{2}=\right.$ $0.204, p=.651)$. Similarly, the effects of treatment on neurocognitive performance did not differ between those studies that did $(\mathrm{g}=0.161, p=.004)$ or did not $(\mathrm{g}=0.166, p=.087)$ utilize ITT analyses $\left(\mathrm{Q}_{2}=0.002, p=.964\right)$.

\section{DISCUSSION}

Results indicate that aerobic exercise training confers modest improvements in neurocognitive function among healthy older adults, including improvements in attention and processing speed, executive function, and memory. Aerobic exercise did not seem to benefit working memory, however. Moderator analyses demonstrated that studies utilizing combined aerobic exercise and strength training interventions improved attention and processing speed and working memory to a greater extent than aerobic exercise alone. In addition, we found preliminary evidence that trials among individuals with MCI may be associated with greater improvements in memory relative to those among noncognitively compromised samples. In contrast, neither training characteristics, such as study duration and intensity, nor methodological quality were associated with differential improvements in neurocognition.

Although previous meta-analytic reviews have reported that exercise may improve neurocognitive performance $(8-12,32)$, ours is one of the largest reviews to date demonstrating that aerobic exercise improves neurocognition among nondemented adults and the first to show that physical activity may enhance memory performance among individuals with MCI, a group at elevated risk for Alzheimer's disease (24). Several previous meta-analytic studies have examined the relationship between physical activity and cognitive function $(8-12,32)$. Colcombe and Kramer (9) reported that RCTs of exercise are associated with clinically meaningful improvements in executive function, processing speed, memory, and motor function. Our findings showed markedly weaker effects relative to this review, most likely as a result of excluding two decidedly positive studies trials included in the meta-analysis of Colcombe and Kramer $(35,36)$ which, on closer examination, were not truly RCTs. In a Cochrane review, Angevaren and colleagues (11) concluded that, 
although RCTs of aerobic exercise among individuals without cognitive impairment were associated with modest improvements in attentional processes, cognitive speed, and motor function, the existing data were insufficient to show that improvements in cognition were attributable to changes in cardiovascular fitness. Similarly, Etnier and colleagues (32) have demonstrated that, although higher levels of fitness were associated with better neurocognitive performance among cross-sectional study designs, studies examining pre and post comparisons found that larger gains in aerobic fitness were associated with lesser improvements in cognitive performance (32). Etnier and colleagues (8) have also noted that methodological limitations contributed to significant variability in treatment effects, with higher-quality studies tending to show smaller effects, and studies with the highest-quality rating demonstrating no effect of exercise on neurocognition. Most recently, van Uffelen and colleagues (12) reported that physical activity interventions among individuals without cognitive decline, on average, tended to report improved neurocognitive function. However, van Uffelen and colleagues (12) did not attempt to statistically combine treatment ESs across studies, reported that the majority of existing trials examining this question have failed to demonstrate a treatment benefit, and found that the extant literature is marked by a lack of high-quality studies. The present analyses address many of the issues raised by this previous review by including several large, high-quality RCTs not previously incorporated in systematic literature syntheses $(30,31,33,34)$.

The finding that exercise may produce larger improvements in memory for individuals with MCI than other patient groups is novel and warrants further investigation, although this must be viewed as preliminary. Although Heyn and colleagues (10) demonstrated that physical activity is associated with improvements in mental status among individuals with dementia, the majority of these trials were conducted among institutionalized adults with dementia and utilized balance and isometric exercises and did not examine the effects of aerobic exercise, specifically, on neurocognition. The finding that aerobic exercise improves memory is consistent with several animal studies, which have indicated that physical activity increases brain-derived neurotrophic factor expression (80) in the hippocampus and perihippocampal structures $(81,82)$. In an important examination of mediators, Pereira and colleagues (83) demonstrated that increased brain-derived neurotrophic factor in the dentate gyrus, an area of the brain proximal to the hippocampus, was associated with dose-response improvements in memory performance among younger adults participating in an exercise intervention. In addition to plausible neurotrophic mediators, it is also possible that individual differences influenced the present findings. For example, it is possible that the MCI samples in our study were composed of a greater number of individuals with the apolipoprotein $E$ type 4 allelic genotype, which has been associated with an increased risk for incident MCI and Alzheimer's disease (84). In addition, evidence (85-87) suggested that individuals with this genotype may exhibit relatively greater neurocog- nitive improvements with physical activity compared with healthy, older adults.

The finding that aerobic exercise alone did not improve working memory performance is an interesting and unpredicted finding. Although it is unclear why aerobic exercise improved other cognitive functions but did not seem to benefit working memory, this finding is consistent with previous brain imaging studies of aerobic exercise. Previous studies have demonstrated that cerebral alterations associated with exercise are preferentially in the perihippocampal region (83), anterior white matter tracks (88), and anterior cingulate (89). Although there is substantial overlap in the brain circuitry for carrying out complex cognitive processes, such as working memory, no imaging studies have demonstrated volumetric changes in the dorsolateral prefrontal cortex, which is primarily subserved by white matter projections from the corpus callosum and most consistently associated with working memory performance $(90,91)$. The finding that combined aerobic exercise and strength training interventions improved attention and working memory to a greater extent than aerobic exercise alone is consistent with previous reviews (9), as well as mechanistic studies demonstrating that strength training may improve neurocognitive function by increasing insulin growth factor, which has been implicated as a mediator of the exercise and neurocognition relationship (92-94). It is also possible that interventions utilizing both aerobic exercise and strength training were more effective in reducing cerebrovascular risk factors (e.g., high blood pressure) (95) and improving aerobic fitness relative to aerobic training alone (96). These improvements in cardiovascular function may reduce the white matter degradation and cerebral ischemia that often result from these conditions (97-99). Alternatively, it is also possible that combined interventions may result in greater improvements in vascular health (100) and basal levels of inflammation $(101,102)$, although these relationships have yet to be investigated.

The present meta-analysis has several limitations. First, the literature is marked by a lack of high-quality trials examining the effects of aerobic exercise on cognitive end points. Trials included in our analyses differed substantially in their use of blinded evaluations, ITT analyses, and clinically validated cognitive assessment tools. Second, RCTs are limited by logistical constraints in their ability to sustain interventions over prolonged periods of time. Accordingly, the majority of studies examining cognitive end points have done so after several months of aerobic training $(79,103)$ or, in some instances, incorporated follow-ups several years later $(33,104)$. There are limited data regarding how physical activity sustained over the course of several years may affect cognitive end points $(2,105)$, despite observational data indicating that physical activity and cardiovascular health may take years to affect brain health $(106,107)$. In addition, RCTs that have examined the neurocognitive effects of aerobic exercise over an extended time period have demonstrated greater improvements in memory over longer follow-up periods $(30,73)$. Third, the majority of extant studies have utilized interven- 
tions with frequency and intensity prescribed in accordance with the American Heart Association recommendations for cardiac rehabilitation (i.e., heart rates at $70 \%$ peak oxygen consumption three times per week). It is, therefore, possible that there was not enough of a range in exercise prescriptions to observe an effect on neurocognition. Finally, there is a lack of consensus as to which neurocognitive measures are most appropriate to examine changes in neurocognitive function associated with exercise. As shown in Table 2, there is substantial heterogeneity in treatment effects among neurocognitive measures. Accordingly, future studies would benefit from the identification of a standardized neurocognitive battery with the appropriate psychometric characteristics to examine neurocognitive measures associated with aerobic exercise.

In conclusion, aerobic exercise training results in modest improvements in cognitive performance among nondemented adults. Trials utilizing longer interventions were associated with greater gains in attention and processing speed, whereas trials conducted among individuals with MCI tended to demonstrate greater improvements in memory relative to non-MCI samples. Additional randomized trials are needed with larger samples, more extensive follow-up periods, appropriate controls, and more extensive measurement of potential mediators of cognitive change. Accordingly, future studies would benefit from the assessment of subclinical vascular health as a potential mediator of the exercise and neurocognition relationship, as this has been associated with improvements in aerobic capacity (100) and neurocognitive performance in other samples $(108,109)$. Future studies should also collect functional magnetic resonance imaging or diffusion tensor imaging measures to track cerebral alterations post exercise, as several previous studies have demonstrated that exercise and improved fitness may increase cerebral blood flow (16) and alter blood oxygen level dependent response patterns to cognitive tasks (89), as well as improve structural brain health, such as by increasing white (88) and gray matter integrity (22) and brain volume (88). Finally, more rigorous studies should examine the effects of aerobic exercise training among individuals with MCI to determine whether this is a plausible strategy to delay or prevent incident dementia (101).

\section{REFERENCES}

1. Laurin D, Verreault R, Lindsay J, MacPherson K, Rockwood K. Physical activity and risk of cognitive impairment and dementia in elderly persons. Arch Neurol 2001;58(3):498-504.

2. Andel R, Crowe M, Pedersen NL, Fratiglioni L, Johansson B, Gatz M. Physical exercise at midlife and risk of dementia three decades later: a population-based study of Swedish twins. J Gerontol A Biol Sci Med Sci 2008;63(1):62-6.

3. Bassuk SS, Wypij D, Berkman LF. Cognitive impairment and mortality in the community-dwelling elderly. Am J Epidemiol 2000;151(7): $676-88$.

4. Fried LP, Kronmal RA, Newman AB, Bild DE, Mittelmark MB, Polak JF, Robbins JA, Gardin JM. Risk factors for 5-year mortality in older adults: the Cardiovascular Health Study. JAMA 1998;279(8):585-92.

5. Kuh D, Richards M, Hardy R, Butterworth S, Wadsworth ME. Childhood cognitive ability and deaths up until middle age: a post-war birth cohort study. Int J Epidemiol 2004;33(2):408-13.

6. Smits CH, Deeg DJ, Kriegsman DM, Schmand B. Cognitive functioning and health as determinants of mortality in an older population. Am J Epidemiol 1999;150(9):978-86.

7. Whalley LJ, Deary IJ. Longitudinal cohort study of childhood IQ and survival up to age 76. BMJ 2001;322(7290):819.

8. Etnier JL, Salazar W, Landers DM, Petruzzello SJ, Han M, Nowell P. The influence of physical fitness and exercise upon cognitive functioning: a meta-analysis. Journal of Sport \& Exercise Psychology 1997;19:249-77.

9. Colcombe S, Kramer AF. Fitness effects on the cognitive function of older adults: a meta-analytic study. Psychol Sci 2003;14(2):125-30.

10. Heyn P, Abreu BC, Ottenbacher KJ. The effects of exercise training on elderly persons with cognitive impairment and dementia: a metaanalysis. Arch Phys Med Rehabil 2004;85(10):1694-704.

11. Angevaren M, Aufdemkampe G, Verhaar HJ, Aleman A, Vanhees L. Physical activity and enhanced fitness to improve cognitive function in older people without known cognitive impairment. Cochrane Database Syst Rev 2008;(2):CD005381.

12. van Uffelen JG, Chin APM, Hopman-Rock M, van MW. The effects of exercise on cognition in older adults with and without cognitive decline: a systematic review. Clin J Sport Med 2008;18(6):486-500.

13. McAuley E, Kramer AF, Colcombe SJ. Cardiovascular fitness and neurocognitive function in older adults: a brief review. Brain Behav Immun 2004;18(3):214-20.

14. Hillman CH, Motl RW, Pontifex MB, Posthuma D, Stubbe JH, Boomsma DI, de Geus EJ. Physical activity and cognitive function in a cross-section of younger and older community-dwelling individuals. Health Psychol 2006;25(6):678-87.

15. Blomquist KB, Danner F. Effects of physical conditioning on information-processing efficiency. Percept Mot Skills 1987;65(1):175-86.

16. Brown AD, McMorris CA, Longman RS, Leigh R, Hill MD, Friedenreich CM, Poulin MJ. Effects of cardiorespiratory fitness and cerebral blood flow on cognitive outcomes in older women. Neurobiol Aging 2008.

17. Chodzko-Zajko WJ, Moore KA. Physical fitness and cognitive functioning in aging. Exerc Sport Sci Rev 1994;22:195-220.

18. Chodzko-Zajko WJ. Physical fitness, cognitive performance, and aging. Med Sci Sports Exerc 1991;23(7):868-72.

19. Clarkson-Smith L, Hartley AA. Relationships between physical exercise and cognitive abilities in older adults. Psychol Aging 1989;4(2):183-9.

20. Elsayed M, Ismail AH, Young RJ. Intellectual differences of adult men related to age and physical fitness before and after an exercise program. J Gerontol 1980;35(3):383-7.

21. Etnier J, Johnston R, Dagenbach D, Pollard RJ, Rejeski WJ, Berry M. The relationships among pulmonary function, aerobic fitness, and $\operatorname{cog}$ nitive functioning in older COPD patients. Chest 1999;116(4):953-60.

22. Gordon BA, Rykhlevskaia EI, Brumback CR, Lee Y, Elavsky S, Konopack JF, McAuley E, Kramer AF, Colcombe S, Gratton G, Fabiani M. Neuroanatomical correlates of aging, cardiopulmonary fitness level, and education. Psychophysiology 2008;45(5):825-38.

23. Weuve J, Kang JH, Manson JE, Breteler MM, Ware JH, Grodstein F. Physical activity, including walking, and cognitive function in older women. JAMA 2004;292(12):1454-61.

24. Larrieu S, Letenneur L, Orgogozo JM, Fabrigoule C, Amieva H, Le CN, Barberger-Gateau P, Dartigues JF. Incidence and outcome of mild cognitive impairment in a population-based prospective cohort. Neurology 2002;59(10):1594-9.

25. Podewils LJ, Guallar E, Kuller LH, Fried LP, Lopez OL, Carlson M, Lyketsos CG. Physical activity, APOE genotype, and dementia risk: findings from the Cardiovascular Health Cognition Study. Am J Epidemiol 2005;161(7):639-51.

26. Singh-Manoux A, Hillsdon M, Brunner E, Marmot M. Effects of physical activity on cognitive functioning in middle age: evidence from the Whitehall II prospective cohort study. Am J Public Health 2005;95(12): 2252-8.

27. Yaffe K, Blackwell T, Gore R, Sands L, Reus V, Browner WS. Depressive symptoms and cognitive decline in nondemented elderly women: a prospective study. Arch Gen Psychiatry 1999;56(5):425-30.

28. Yaffe K, Barnes D, Nevitt M, Lui LY, Covinsky K. A prospective study of physical activity and cognitive decline in elderly women: women who walk. Arch Intern Med 2001;161(14):1703-8.

29. Okumiya K, Matsubayashi K, Wada T, Kimura S, Doi Y, Ozawa T. Effects of exercise on neurobehavioral function in community-dwelling older people more than 75 years of age. J Am Geriatr Soc 1996;44(5): $569-72$. 
30. Lautenschlager NT, Cox KL, Flicker L, Foster JK, van Bockxmeer FM, Xiao J, Greenop KR, Almeida OP. Effect of physical activity on cognitive function in older adults at risk for Alzheimer disease: a randomized trial. JAMA 2008;300(9):1027-37.

31. Hoffman BM, Blumenthal JA, Babyak MA, Smith PJ, Rogers SD, Doraiswamy PM, Sherwood A. Exercise fails to improve neurocognition in depressed middle-aged and older adults. Med Sci Sports Exerc 2008;40(7):1344-52.

32. Etnier JL, Nowell PM, Landers DM, Sibley BA. A meta-regression to examine the relationship between aerobic fitness and cognitive performance. Brain Res Brain Res Rev 2006;52(1):119-30.

33. van Uffelen JG, Chinapaw MJ, van MW, Hopman-Rock M. Walking or vitamin $\mathrm{B}$ for cognition in older adults with mild cognitive impairment? A randomised controlled trial. Br J Sports Med 2008;42(5):344-51.

34. Williamson JD, Espeland M, Kritchevsky SB, Newman AB, King AC, Pahor M, Guralnik JM, Pruitt LA, Miller ME. Changes in cognitive function in a randomized trial of physical activity: results of the lifestyle interventions and independence for elders pilot study. J Gerontol A Biol Sci Med Sci 2009;64(6):688-94.

35. Rikli RE, Edwards DJ. Effects of a three-year exercise program on motor function and cognitive processing speed in older women. Res Q Exerc Sport 1991;62(1):61-7.

36. Dustman RE, Ruhling RO, Russell EM, Shearer DE, Bonekat HW, Shigeoka JW, Wood JS, Bradford DC. Aerobic exercise training and improved neuropsychological function of older individuals. Neurobiol Aging 1984;5(1):35-42.

37. Tamnes CK, Ostby Y, Fjell AM, Westlye LT, Due-Tonnessen P, Walhovd KB. Brain Maturation in Adolescence and Young Adulthood: Regional Age-Related Changes in Cortical Thickness and White Matter Volume and Microstructure. Cereb Cortex 2009.

38. Hill RD, Storandt M, Malley M. The impact of long-term exercise training on psychological function in older adults. J Gerontol 1993; 48(1):12-7.

39. Hassmen P, Ceci R, Backman L. Exercise for older women: a training method and its influences on physical and cognitive performance. Eur J Appl Physiol Occup Physiol 1992;64(5):460-6.

40. Bastone AC, Jacob FW. Effect of an exercise program on functional performance of institutionalized elderly. J Rehabil Res Dev 2004;41(5): $659-68$.

41. Tanaka K, Quadros AC Jr, Santos RF, Stella F, Gobbi LT, Gobbi S. Benefits of physical exercise on executive functions in older people with Parkinson's disease. Brain Cogn 2009;69(2):435-41.

42. Zlomanczuk P, Milczarek B, Dmitruk K, Sikorski W, Adamczyk W, Zegarski T, Tafil-Klawe M, Chesy G, Klawe JJ, Rakowski A. Improvement in the face/name association performance after three months of physical training in elderly women. J Physiol Pharmacol 2006;57 Suppl $4: 417-24$.

43. Winter B, Breitenstein C, Mooren FC, Voelker K, Fobker M, Lechtermann A, Krueger K, Fromme A, Korsukewitz C, Floel A, Knecht S. High impact running improves learning. Neurobiol Learn Mem 2006.

44. Brisswalter J, Arcelin R, Audiffren M, Delignieres D. Influence of physical exercise on simple reaction time: effect of physical fitness. Percept Mot Skills 1997;85(3 Pt 1):1019-27.

45. Molloy DW, Beerschoten DA, Borrie MJ, Crilly RG, Cape RD. Acute effects of exercise on neuropsychological function in elderly subjects. J Am Geriatr Soc 1988;36(1):29-33.

46. Ploughman M, McCarthy J, Bosse M, Sullivan HJ, Corbett D. Does treadmill exercise improve performance of cognitive or upper-extremity tasks in people with chronic stroke? A randomized cross-over trial. Arch Phys Med Rehabil 2008;89(11):2041-7.

47. Rabadi M, Galgano M, Lynch D, Akerman M, Lesser M, Volpe B. A pilot study of activity-based therapy in the arm motor recovery post stroke: a randomized controlled trial. Clin Rehabil 2008;22(12): 1071-82.

48. Stevenson JS, Topp R. Effects of moderate and low intensity long-term exercise by older adults. Res Nurs Health 1990;13(4):209-18.

49. Cosky AC. The effect of aerobic exercise on fitness status, cognition, and health locus of control in older women. Unpublished Dissertation 1989.

50. Zervas Y, Danis A, Klissouras V. Influence of physical exertion on mental performance with reference to training. Percept Mot Skills 1991; 72(3 Pt 2):1215-21.
51. Evans JJ, Greenfield E, Wilson BA, Bateman A. Walking and talking therapy: improving cognitive-motor dual-tasking in neurological illness. J Int Neuropsychol Soc 2009;15(1):112-20.

52. Cott CA, Dawson P, Sidani S, Wells D. The effects of a walking/talking program on communication, ambulation, and functional status in residents with Alzheimer disease. Alzheimer Dis Assoc Disord 2002;16(2): $81-7$

53. Marmeleira JF, Godinho MB, Fernandes OM. The effects of an exercise program on several abilities associated with driving performance in older adults. Accid Anal Prev 2009;41(1):90-7.

54. Brown AK, Liu-Ambrose T, Tate R, Lord SR. The effect of group-based exercise on cognitive performance and mood in seniors residing in intermediate care and self-care retirement facilities: a randomised controlled trial. Br J Sports Med 2009;43(8):608-14.

55. Liu-Ambrose T, Donaldson MG, Ahamed Y, Graf P, Cook WL, Close J, Lord SR, Khan KM. Otago home-based strength and balance retraining improves executive functioning in older fallers: a randomized controlled trial. J Am Geriatr Soc 2008;56(10):1821-30.

56. Friedman R, Tappen RM. The effect of planned walking on communication in Alzheimer's disease. J Am Geriatr Soc 1991;39(7):650-4.

57. McMurdo ME, Rennie L. A controlled trial of exercise by residents of old people's homes. Age Ageing 1993;22(1):11-5.

58. Molloy DW, Richardson LD, Crilly RG. The effects of a three-month exercise programme on neuropsychological function in elderly institutionalized women: a randomized controlled trial. Age Ageing 1988; 17(5):303-10.

59. Nowalk MP, Prendergast JM, Bayles CM, D’Amico FJ, Colvin GC. A randomized trial of exercise programs among older individuals living in two long-term care facilities: the FallsFREE program. J Am Geriatr Soc 2001;49(7):859-65.

60. Schnelle JF, MacRae PG, Giacobassi K, MacRae HS, Simmons SF, Ouslander JG. Exercise with physically restrained nursing home residents: maximizing benefits of restraint reduction. J Am Geriatr Soc 1996;44(5):507-12.

61. Palleschi L, Vetta F, De GE, Idone G, Sottosanti G, Gianni W, Marigliano V. Effect of aerobic training on the cognitive performance of elderly patients with senile dementia of alzheimer type. Arch Gerontol Geriatr 1996;22 Suppl 1:47-50.

62. Christofoletti G, Oliani MM, Gobbi S, Stella F, Bucken Gobbi LT, Renato CP. A controlled clinical trial on the effects of motor intervention on balance and cognition in institutionalized elderly patients with dementia. Clin Rehabil 2008;22(7):618-26.

63. Mulrow CD, Gerety MB, Kanten D, Cornell JE, DeNino LA, Chiodo L, Aguilar C, O’Neil MB, Rosenberg J, Solis RM. A randomized trial of physical rehabilitation for very frail nursing home residents. JAMA 1994;271(7):519-24.

64. Powell RR. Psychological effects of exercise therapy upon institutionalized geriatric mental patients. J Gerontol 1974;29(2):157-61.

65. McMurdo ME, Rennie LM. Improvements in quadriceps strength with regular seated exercise in the institutionalized elderly. Arch Phys Med Rehabil 1994;75(5):600-3.

66. Hawkins HL, Kramer AF, Capaldi D. Aging, exercise, and attention. Psychol Aging 1992;7(4):643-53.

67. Lezak MD, Howieson DB, Loring DW. Neuropsychological assessment. 4th ed. ed. New York: Oxford University Press; 2004.

68. Anderson JR. Cognitive Psychology and Its Implications. 6th ed. Worth Publishers and W.H. Freeman and Company; 2005.

69. Cohen J. Statistical power analysis for the behavioral sciences. 2nd ed. Hillsdale, NJ: Erlbaum; 1988

70. Schmidt FL, Oh IS, Hayes TL. Fixed- versus random-effects models in meta-analysis: model properties and an empirical comparison of differences in results. Br J Math Stat Psychol 2009;62(Pt 1):97-128.

71. Madden DJ, Blumenthal JA, Allen PA, Emery CF. Improving aerobic capacity in healthy older adults does not necessarily lead to improved cognitive performance. Psychol Aging 1989;4(3):307-20.

72. Blumenthal JA, Emery CF, Madden DJ, George LK, Coleman RE, Riddle MW, McKee DC, Reasoner J, Williams RS. Cardiovascular and behavioral effects of aerobic exercise training in healthy older men and women. J Gerontol 1989;44(5):M147-57.

73. Blumenthal JA, Emery CF, Madden DJ, Schniebolk S, Walsh-Riddle M, George LK, McKee DC, Higginbotham MB, Cobb FR, Coleman RE. Long-term effects of exercise on psychological functioning in older men and women. J Gerontol 1991;46(6):352-61. 
74. Kramer AF, Hahn S, Cohen NJ, Banich MT, McAuley E, Harrison CR, Chason J, Vakil E, Bardell L, Boileau RA, Colcombe A. Ageing, fitness and neurocognitive function. Nature 1999;400(6743):418-9.

75. Kramer AF, Hahn S, McAuley E, Cohen NJ, Banich MT, Harrison C, Chason J, Boileau RA, Bardell L, Colcombe A, Vakil E. Exercise, aging, and cognition: healthy body, healthy mind? In: Rogers WA, Fisk $\mathrm{AD}$, editors. Human Factors Interventions for the Health Care of Older Adults. Mahwah, NJ: Erlbaum; 2002. p. 91-120.

76. Emery CF, Shermer RL, Hauck ER, Hsiao ET, MacIntyre NR. Cognitive and psychological outcomes of exercise in a 1-year follow-up study of patients with chronic obstructive pulmonary disease. Health Psychol 2003;22(6):598-604.

77. Hedges LV, Pigott TD. The power of statistical tests for moderators in meta-analysis. Psychol Methods 2004;9(4):426-45.

78. Greenhouse JB, Iyengar S. Sensitivity Analysis and Diagnostics. In: Cooper H, Hedges LV, editors. The Handbook of Research Synthesis. New York: Russell Sage Foundation; 1994. p. 383-98.

79. Scherder EJ, Van PJ, Deijen JB, Van Der KS, Orlebeke JF, Burgers I, Devriese PP, Swaab DF, Sergeant JA. Physical activity and executive functions in the elderly with mild cognitive impairment. Aging Ment Health 2005;9(3):272-80.

80. Gomez-Pinilla F, Ying Z, Roy RR, Molteni R, Edgerton VR. Voluntary exercise induces a BDNF-mediated mechanism that promotes neuroplasticity. J Neurophysiol 2002;88(5):2187-95.

81. Russo-Neustadt AA, Alejandre H, Garcia C, Ivy AS, Chen MJ. Hippocampal brain-derived neurotrophic factor expression following treatment with reboxetine, citalopram, and physical exercise. Neuropsychopharmacology 2004;29(12):2189-99.

82. Vaynman S, Ying Z, Gomez-Pinilla F. Hippocampal BDNF mediates the efficacy of exercise on synaptic plasticity and cognition. Eur J Neurosci 2004;20(10):2580-90.

83. Pereira AC, Huddleston DE, Brickman AM, Sosunov AA, Hen R, McKhann GM, Sloan R, Gage FH, Brown TR, Small SA. An in vivo correlate of exercise-induced neurogenesis in the adult dentate gyrus. Proc Natl Acad Sci U S A 2007;104(13):5638-43.

84. Bennett DA, Wilson RS, Schneider JA, Evans DA, Aggarwal NT, Arnold SE, Cochran EJ, Berry-Kravis E, Bienias JL. Apolipoprotein E epsilon4 allele, AD pathology, and the clinical expression of Alzheimer's disease. Neurology 2003;60(2):246-52.

85. Schuit AJ, Feskens EJ, Launer LJ, Kromhout D. Physical activity and cognitive decline, the role of the apolipoprotein e4 allele. Med Sci Sports Exerc 2001;33(5):772-7.

86. Lindsay J, Laurin D, Verreault R, Hebert R, Helliwell B, Hill GB, McDowell I. Risk factors for Alzheimer's disease: a prospective analysis from the Canadian Study of Health and Aging. Am J Epidemiol 2002;156(5):445-53.

87. Etnier JL, Caselli RJ, Reiman EM, Alexander GE, Sibley BA, Tessier D, McLemore EC. Cognitive performance in older women relative to ApoE-epsilon4 genotype and aerobic fitness. Med Sci Sports Exerc 2007;39(1):199-207.

88. Colcombe SJ, Erickson KI, Scalf PE, Kim JS, Prakash R, McAuley E, Elavsky S, Marquez DX, Hu L, Kramer AF. Aerobic exercise training increases brain volume in aging humans. J Gerontol A Biol Sci Med Sci 2006;61(11):1166-70.

89. Colcombe SJ, Kramer AF, Erickson KI, Scalf P, McAuley E, Cohen NJ, Webb A, Jerome GJ, Marquez DX, Elavsky S. Cardiovascular fitness, cortical plasticity, and aging. Proc Natl Acad Sci U S A 2004;101(9): $3316-21$.

90. Petrides M. The role of the mid-dorsolateral prefrontal cortex in working memory. Exp Brain Res 2000;133(1):44-54.

91. Macdonald AW III, Cohen JD, Stenger VA, Carter CS. Dissociating the role of the dorsolateral prefrontal and anterior cingulate cortex in cognitive control. Science 2000;288(5472):1835-8.

92. Aberg ND, Brywe KG, Isgaard J. Aspects of growth hormone and insulin-like growth factor-I related to neuroprotection, regeneration, and functional plasticity in the adult brain. ScientificWorldJournal 2006;6: 53-80.

93. Ding Q, Vaynman S, Akhavan M, Ying Z, Gomez-Pinilla F. Insulin-like growth factor I interfaces with brain-derived neurotrophic factormediated synaptic plasticity to modulate aspects of exercise-induced cognitive function. Neuroscience 2006;140(3):823-33.

94. Liu-Ambrose T, Donaldson MG. Exercise and cognition in older adults: is there a role for resistance training programmes? Br J Sports Med 2009;43(1):25-7.
95. Stewart KJ, Bacher AC, Turner KL, Fleg JL, Hees PS, Shapiro EP, Tayback M, Ouyang P. Effect of exercise on blood pressure in older persons: a randomized controlled trial. Arch Intern Med 2005;165(7): 756-62.

96. Marzolini S, Oh PI, Thomas SG, Goodman JM. Aerobic and resistance training in coronary disease: single versus multiple sets. Med Sci Sports Exerc 2008;40(9):1557-64.

97. Knopman D, Boland LL, Mosley T, Howard G, Liao D, Szklo M, McGovern P, Folsom AR. Cardiovascular risk factors and cognitive decline in middle-aged adults. Neurology 2001;56(1):42-8.

98. Tzourio C, Dufouil C, Ducimetiere P, Alperovitch A. Cognitive decline in individuals with high blood pressure: a longitudinal study in the elderly. EVA Study Group. Epidemiology of Vascular Aging. Neurology 1999;53(9):1948-52.

99. Whelton SP, Chin A, Xin X, He J. Effect of aerobic exercise on blood pressure: a meta-analysis of randomized, controlled trials. Ann Intern Med 2002;136(7):493-503.

100. Green DJ, Maiorana A, O'Driscoll G, Taylor R. Effect of exercise training on endothelium-derived nitric oxide function in humans. J Physiol 2004;561(Pt 1):1-25.

101. Barnes DE, Whitmer RA, Yaffe K. Physical activity and dementia: The need for prevention trials. Exerc Sport Sci Rev 2007;35(1):24-9.

102. Mora S, Cook N, Buring JE, Ridker PM, Lee IM. Physical activity and reduced risk of cardiovascular events: potential mediating mechanisms. Circulation 2007;116(19):2110-8.

103. Bakken RC, Carey JR, Di Fabio RP, Erlandson TJ, Hake JL, Intihar TW. Effect of aerobic exercise on tracking performance in elderly people: a pilot study. Phys Ther 2001;81(12):1870-9.

104. Williams P, Lord SR. Effects of group exercise on cognitive functioning and mood in older women. Aust N Z J Public Health 1997;21(1):45-52.

105. Richards M, Hardy R, Wadsworth ME. Does active leisure protect cognition? Evidence from a national birth cohort. Soc Sci Med 2003; 56(4):785-92.

106. Beason-Held LL, Moghekar A, Zonderman AB, Kraut MA, Resnick SM. Longitudinal changes in cerebral blood flow in the older hypertensive brain. Stroke 2007;38(6):1766-73.

107. Rovio S, Kareholt I, Helkala EL, Viitanen M, Winblad B, Tuomilehto J, Soininen H, Nissinen A, Kivipelto M. Leisure-time physical activity at midlife and the risk of dementia and Alzheimer's disease. Lancet Neurol 2005;4(11):705-11.

108. Smith PJ, Blumenthal JA, Babyak MA, Hoffman BM, Doraiswamy PM, Waugh R, Hinderliter A, Sherwood A. Cerebrovascular risk factors, vascular disease, and neuropsychological outcomes in adults with major depression. Psychosom Med 2007;69(6):578-86.

109. Cohen RA, Poppas A, Forman DE, Hoth KF, Haley AP, Gunstad J, Jefferson AL, Tate DF, Paul RH, Sweet LH, Ono M, Jerskey BA, Gerhard-Herman M. Vascular and cognitive functions associated with cardiovascular disease in the elderly. J Clin Exp Neuropsychol 2008; $1-15$.

110. Emery CF, Gatz M. Psychological and cognitive effects of an exercise program for community-residing older adults. Gerontologist 1990; 30(2):184-8.

111. Emery CF, Schein RL, Hauck ER, MacIntyre NR. Psychological and cognitive outcomes of a randomized trial of exercise among patients with chronic obstructive pulmonary disease. Health Psychol 1998;17(3): 232-40.

112. Fabre C, Chamari K, Mucci P, Masse-Biron J, Prefaut C. Improvement of cognitive function by mental and/or individualized aerobic training in healthy elderly subjects. Int J Sports Med 2002;23(6):415-21.

113. Khatri P, Blumenthal JA, Babyak MA, Craighead WE, Herman S, Baldewicz T, Madden DJ, Doraiswamy PM, Waugh R, Krishnan KR. Effects of exercise training on cognitive functioning among depressed older men and women. J Aging Phys Act 2001;9:43-57.

114. Masley SC, Weaver W, Peri G, Phillips SE. Efficacy of lifestyle changes in modifying practical markers of wellness and aging. Altern Ther Health Med 2008;14(2):24-9.

115. Moul JL, Goldman B, Warren B. Physical activity and cognitive performance in the older population. J Aging Phys Act 1995;3(2):135-45.

116. Munguia-Izquierdo D, Legaz-Arrese A. Assessment of the effects of aquatic therapy on global symptomatology in patients with fibromyalgia syndrome: a randomized controlled trial. Arch Phys Med Rehabil 2008; 89(12):2250-7.

117. Oken BS, Kishiyama S, Zajdel D, Bourdette D, Carlsen J, Haas M, 


\section{P. J. SMITH et al.}

Hugos C, Kraemer DF, Lawrence J, Mass M. Randomized controlled trial of yoga and exercise in multiple sclerosis. Neurology 2004;62(11): 2058-64.

118. Oken BS, Zajdel D, Kishiyama S, Flegal K, Dehen C, Haas M, Kraemer DF, Lawrence J, Leyva J. Randomized, controlled, six-month trial of yoga in healthy seniors: effects on cognition and quality of life. Altern Ther Health Med 2006;12(1):40-7.

119. Panton LB, Graves JE, Pollock ML, Hagberg JM, Chen W. Effect of aerobic and resistance training on fractionated reaction time and speed of movement. J Gerontol 1990;45(1):M26-31.

120. Pierce TW, Madden DJ, Siegel WC, Blumenthal JA. Effects of aerobic exercise on cognitive and psychosocial functioning in patients with mild hypertension. Health Psychol 1993;12(4):286-91.

121. Perri S, Templer DI. The effects of an aerobic exercise program on psychological variables in older adults. Int J Aging Hum Dev 1984; 20(3):167-72.
122. Russell EM. The effects of an aerobic conditioning program on reaction times of older sedentary adults. Unpublished Dissertation, 167-172. 1984. University of Utah, Salt Lake City. Ref Type: Generic

123. Smiley-Oyen AL, Lowry KA, Francois SJ, Kohut ML, Ekkekakis P. Exercise, fitness, and neurocognitive function in older adults: the "selective improvement" and "cardiovascular fitness" hypotheses. Ann Behav Med 2008;36(3):280-91.

124. Stroth S, Hille K, Spitzer M, Reinhardt R. Aerobic endurance exercise benefits memory and affect in young adults. Neuropsychol Rehabil 2009;19(2):223-43.

125. Wallman KE, Morton AR, Goodman C, Grove R, Guilfoyle AM. Randomised controlled trial of graded exercise in chronic fatigue syndrome. Med J Aust 2004;180(9):444-8.

126. Whitehurst M. Reaction time unchanged in older women following aerobic training. Percept Mot Skills 1991;72(1):251-6. 